\title{
Synthesis and antimicrobial activities of gold(I) sulfanylcarboxylates
}

\author{
Elena Barreiro • José S. Casas • María D. Couce • \\ Agustín Sánchez • Rafael Seoane • \\ Antonio Perez-Estévez • José Sordo
}

Published online: 26 January 2012

(C) The Author(s) 2012. This article is published with open access at Springerlink.com

\begin{abstract}
Reaction of $\mathrm{NaAuCl}_{4} \cdot \mathrm{H}_{2} \mathrm{O}$ and thiodiglycol (1:3 molar ratio) with 3-(aryl)-2-sulfanylpropenoic acids, $\mathrm{H}_{2} x$ spa $=[x: p=3$-phenyl-, $f=3$-(2-furyl)-, $t=3$-(2-thienyl)-, $o$ - $p y=3$-(2-pyridyl)-, $C l p=3$-(2-chlorophenyl)-, $-o-m p=3$ (2-methoxyphenyl)-, - $p-m p=3-(4-m e t h o x y p h e n y l)-,-o-$ $h p=3$-(2-hydroxyphenyl)-, $-p-h p=3$-(4-hydroxyphenyl)-, diBr-o-hp=3-(3,5-dibromo-2-hydroxyphenyl)] and 2cyclopentylidene-2-sulfanylacetic acid $\left(\mathrm{H}_{2} \mathrm{cpa}\right)$ in a $1: 1$ metal/ligand molar ratio gave compounds of the type $[\mathrm{Au}(\mathrm{H} x \mathrm{spa})]$ or $[\mathrm{Au}(\mathrm{Hcpa})]$. These compounds were reacted with diisopropylamine to afford $[\mathrm{HQ}][\mathrm{Au}(x \mathrm{spa})]$ or $[\mathrm{HQ}][\mathrm{Au}(\mathrm{cpa})](\mathrm{HQ}=$ diisopropylammonium $)$ and with $\mathrm{NaOH}$ to afford $\mathrm{Na}[\mathrm{Au}(x \mathrm{spa})] \cdot \mathrm{H}_{2} \mathrm{O}$ and $\mathrm{Na}[\mathrm{Au}(\mathrm{cpa})] \cdot \mathrm{H}_{2} \mathrm{O}$. All of the new compounds were isolated and characterised by IR and ${ }^{1} \mathrm{H}$ and ${ }^{13} \mathrm{C}$ NMR spectroscopy. The antimicrobial activities of the complexes against Escherichia coli, Staphylococcus aureus, Bacillus subtilis, Candida albicans, Pseudomonas aeruginosa and carbapenem-resistant $P$. aeruginosa were evaluated and compared to those of the equivalent silver(I) complexes. The comparison shows that the gold compounds generally show better activity than the silver analogues against $S$. aureus and
\end{abstract}

E. Barreiro · J. S. Casas · A. Sánchez $\cdot$ J. Sordo $(\square)$

Departamento de Química Inorgánica, Facultade de Farmacia, Universidade de Santiago de Compostela,

15782 Santiago de Compostela Galicia, Spain

e-mail: jose.sordo@usc.es

M. D. Couce

Departamento de Química Inorgánica, Facultade de Ciencias, Universidade de Vigo,

36310 Vigo Galicia, Spain

R. Seoane $\cdot$ A. Perez-Estévez

Departamento de Microbiología, Facultade de Medicina,

Universidade de Santiago de Compostela,

15782 Santiago de Compostela Galicia, Spain
B. subtilis, but low sensitivity against $E$. coli, P. aeruginosa and $C$. albicans, suggesting a different mode of antimicrobial action for equivalent silver and gold compounds.

Keywords Gold(I) complexes · Sulfanylpropenoic acids .

2-Cyclopentylidene-2-sulfanylacetic acid .

Antimicrobial studies

\section{Introduction}

Silver(I) and gold(I) compounds present a variety of biological activities and have various medicinal uses, the study of which has increased in recent years. Silver compounds have mainly been studied for their widely known antibacterial effect; in fact, silver nitrate and certain silver complexes are still used against local infections [1-3]. From this perspective, compounds with Ag kernels have recently been prepared and studied, including examples containing $\mathrm{Ag}-\mathrm{N}$ [4-7], $\mathrm{Ag}-\mathrm{O}$ [8-10] and Ag-S [11-15] bonds and some with other additional bonds. In previous papers, we have contributed [14, 15] to the study of $\mathrm{Ag}-\mathrm{S}$ by preparing compounds of the type $[\mathrm{Ag}$ $(\mathrm{HL})],\left[\mathrm{Ag}_{2}(\mathrm{~L})\right],[\mathrm{HQ}][\mathrm{Ag}(\mathrm{L})](\mathrm{HQ}=$ diisopropylammonium $)$ and $\mathrm{Na}[\mathrm{Ag}(\mathrm{L})] \cdot \mathrm{xH}_{2} \mathrm{O}$, where $\mathrm{H}_{2} \mathrm{~L}$ is a 3-(aryl)-2-sulfanylpropenoic acid or 2-cyclopentylidene-2-sulfanylacetic acid, which can be present as a mono- or bideprotonated system in the complexes. The last two classes of compounds, especially the latter, show activity against certain Gram-positive and Gramnegative bacteria, and also against the yeast Candida albicans; this activity is similar to that shown by other compounds with $\mathrm{Ag}-\mathrm{N}$ and/or $\mathrm{Ag}-\mathrm{O}$ bonds except in the case of Escherichia coli, for which they show only low activity.

Studies on gold complexes have mainly focused on antiarthritic properties [16-21], but growing interest is evident in antitumoral [22-24], antiparasitic [25] and antibacterial 
activities. In this last field, the compounds under investigation include a major collection of the type $\mathrm{R}_{3} \mathrm{PAuL}$, in which the gold atom is coordinated to a phosphine ligand and $\mathrm{L}$ is an O- [26, 27], N- [28, 29], Cl- [30] or S-[29, 31-35] donor ligand. Complexes including S-donor ligands as the only ligand include the anionic complexes $\mathrm{H}\left[\mathrm{Au}(\mathrm{Hmna})_{2}\right]$, $\mathrm{Na}_{3}\left[\mathrm{Au}(\mathrm{mna})_{2}\right]\left(\mathrm{H}_{2} \mathrm{mna}=2\right.$-mercaptonicotinic acid $)$ [13] and the cationic complexes $\left[\mathrm{Au}(\mathrm{L})_{2}\right]\left(\mathrm{NO}_{3}\right)_{3}(\mathrm{~L}=1$-[2-(acridin-9-ylamino)ethyl]-1,3-dimethylthiourea) [34]. For the latter ligand, compounds of the type $[\mathrm{Au}(\mathrm{L})] \mathrm{Br},[\mathrm{Au}(\mathrm{L})]$ $\mathrm{SCN}$ and $[\mathrm{Au}(\mathrm{L})] \mathrm{Cl}$ were also prepared [34] and the coexistence of an S-donor ligand and a $\mathrm{Cl}$ ligand was also described for [LAuCl] (where $\mathrm{L}=2,3$-diphenyl-1,3,4thiadiazolium-5-thiolato- $\mathrm{S}_{\text {exo }}$ ) [36].

The activity of some of these complexes is significant against some bacteria or mycobacteria; however, there is a limited number of gold(I) complexes of this class that have been studied and a broad comparative study with equivalent silver(I) complexes having similar ligand/metal stoichiometry has not been carried out.

As mentioned above, we have previously prepared [14, 15] several silver(I) complexes containing a variety of sulfanylcarboxylate ligands, thus enabling the aforementioned comparative study. In order to carry out this comparison, we have prepared and characterised equivalent gold(I) complexes of the type $[\mathrm{Au}(\mathrm{HL})],[\mathrm{HQ}][\mathrm{Au}(\mathrm{L})](\mathrm{HQ}=$ diisopropylammonium) and $\mathrm{Na}[\mathrm{Au}(\mathrm{L})] \cdot \mathrm{H}_{2} \mathrm{O}$. The activities of these complexes were determined and analysed in light of the results obtained for the equivalent silver(I) complexes.

\section{Experimental}

Materials and methods

The 3-(aryl)-2-sulfanylpropenoic acids (Scheme 1) were prepared by condensation of the appropriate aldehyde with rhodanine, subsequent hydrolysis in an alkaline medium and acidification with aqueous $\mathrm{HCl}$. In the preparation of 2cyclopentylidene-2-sulfanylacetic acid, a ketone (cyclopentanone) was used in the condensation reaction instead of an aldehyde [14, 15, 37]. $\mathrm{NaAuCl}_{4} \cdot 2 \mathrm{H}_{2} \mathrm{O}$ (Aldrich), $\mathrm{S}$ $\left(\mathrm{CH}_{2} \mathrm{CH}_{2} \mathrm{OH}\right)_{2}$ (Aldrich), diisopropylamine (Merck) and $\mathrm{NaOH}$ (Probus) were all used as supplied.

Elemental analyses were performed on a Fisons 1108 microanalyser. Melting points were determined with a Büchi apparatus. IR spectra ( $\mathrm{KBr}$ pellets or Nujol mulls) were recorded on a Bruker IFS66V FT-IR spectrophotometer and are reported in the synthesis section using the following abbreviations: $\mathrm{vs}=$ very strong, $\mathrm{s}=$ strong, $\mathrm{m}=$ medium, $\mathrm{w}=$ weak, sh=shoulder, br=broad. ${ }^{1} \mathrm{H},{ }^{13} \mathrm{C}$ and DEPT NMR spectra in solution were recorded in dimethyl sulfoxide (DMSO)- $\mathrm{d}_{6}$ at room temperature on a Bruker AMX 300 spectrometer operating at $300.14 \mathrm{MHz}\left({ }^{1} \mathrm{H}\right)$ and $75.40 \mathrm{MHz}$ $\left({ }^{13} \mathrm{C}\right)$, using 5 -mm o.d. tubes; chemical shifts are reported relative to TMS using the solvent signal $\left({ }^{1} \mathrm{H}=2.50 \mathrm{ppm} ; \delta\right.$ $\left.{ }^{13} \mathrm{C}=39.50 \mathrm{ppm}\right)$ as reference. The splitting of proton resonances in the reported ${ }^{1} \mathrm{H}$ NMR spectra are defined as $\mathrm{s}=$ singlet, $\mathrm{d}=$ doublet, $\mathrm{t}=$ triplet, $\mathrm{m}=$ multiplet, $\mathrm{pst}=$ pseudotriplet and $\mathrm{br}=$ broad. The numbering scheme is shown in Scheme 1.

\section{Antimicrobial activity}

Antibacterial activity was initially assayed by MüllerHinton agar diffusion methods. Compounds 1-11 were suspended in water containing $0.1 \%$ of DMSO, the ligands and compounds 12-22 were dissolved in ethanol and compounds 23-33 were dissolved in water. Paper discs $(5 \mathrm{~mm}$ in diameter) were impregnated with $20 \mu \mathrm{L}$ of a $2 \mathrm{mg} / \mathrm{cm}^{3}$ solution or suspension of the substance to be tested and control discs were impregnated with solvent alone. The discs were then placed on dishes of Müller-Hinton agar inoculated with Staphylococcus aureus (ATCC29213), Bacillus subtilis (ATCC6633), Escherichia coli (ATCC25922), Pseudomonas aeruginosa (ATCC27853) and a carbapenemresistant strain of $P$. aeruginosa (hereafter "resistant $P$. aeruginosa"). After incubation for $24 \mathrm{~h}$ at $37^{\circ} \mathrm{C}$, the diameters of the bacterial growth inhibition zones were measured. All assays were carried out in duplicate. For products that showed activity, serial dilutions in Müller-Hinton broth were used as described in the literature [38] to determine the minimum inhibitory concentration (MIC), which is defined as the lowest concentration of the substance under test that inhibits the visible growth of the test organism when the latter is at optimal concentration. Minimum bactericidal concentration (MCB) was also determined for active compounds. Briefly, after MIC determination ( $24 \mathrm{~h}$ of exposure to compounds) bacterial cultures were subcultured in plates of solid medium without test compounds and incubated for $24 \mathrm{~h}$. MCB was defined as the lowest concentration able to prevent bacterial growth in the first $24 \mathrm{~h}$ after compound removal.

\section{Synthesis}

Complexes of the type $[A u(H L)]$ Complexes 1-11 were prepared by adding a stirred solution of $\mathrm{NaAuCl}_{4} \cdot \mathrm{H}_{2} \mathrm{O}$ and S $\left(\mathrm{CH}_{2} \mathrm{CH}_{2} \mathrm{OH}\right)_{2}$ (thiodiglycol) in a 1:3 molar ratio in water to a solution of the appropriate sulfanylcarboxylic acid (metal/ ligand molar ratio 1:1) in ethanol. The mixture was stirred at room temperature for $1 \mathrm{~h}$ and the resulting solid was filtered off, washed with ethanol, water and ether and dried in vacuo.

[Au(Hpspa)] (1). $\mathrm{H}_{2}$ pspa $(0.07 \mathrm{~g}, 0.38 \mathrm{mmol})$, $\mathrm{NaAuCl}_{4} \cdot 3 \mathrm{H}_{2} \mathrm{O}(0.15 \mathrm{~g}, 0.38 \mathrm{mmol})$, thiodiglycol $\left(0.11 \mathrm{~cm}^{3}\right)$, ethanol $\left(3 \mathrm{~cm}^{3}\right), \mathrm{H}_{2} \mathrm{O}\left(5 \mathrm{~cm}^{3}\right)$, yellow solid. 
Scheme 1 The numbering scheme of 3-(aryl)-

2 -sulfanylpropenoic acids and

2-cyclopentylidene-2-

sulfanylacetic acid and synthesis of complexes
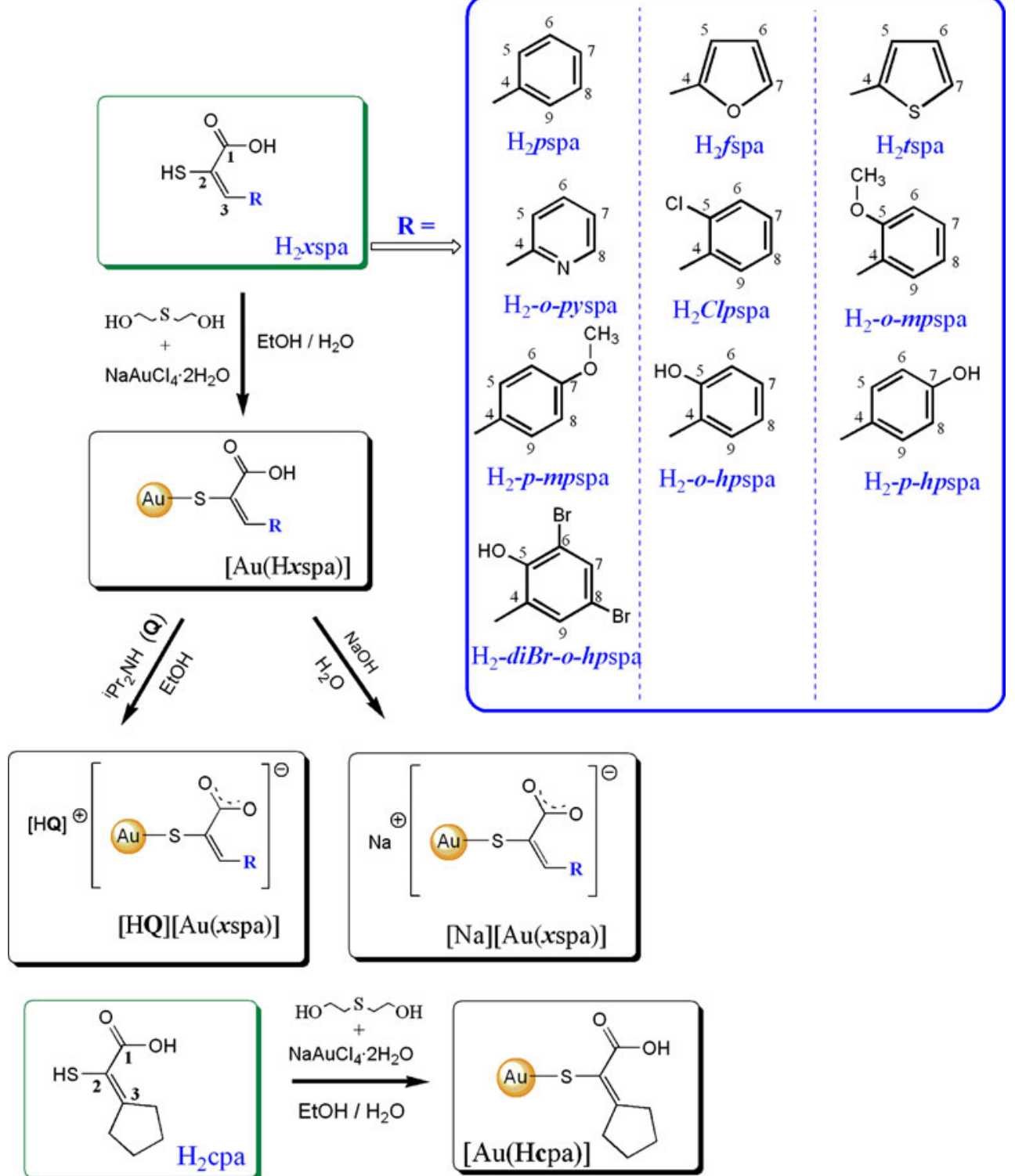

$\int_{0}^{5}$<smiles>Cc1cccs1</smiles>

$\mathrm{H}_{2} t$ spa

$\mathrm{H}_{2}$-o-mpspa

$\mathrm{H}_{2}-p-h p$ spa

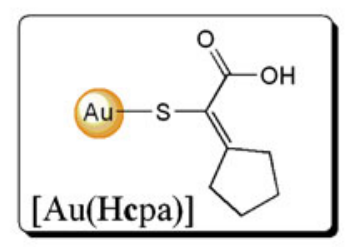

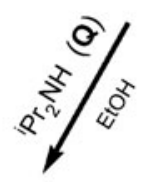
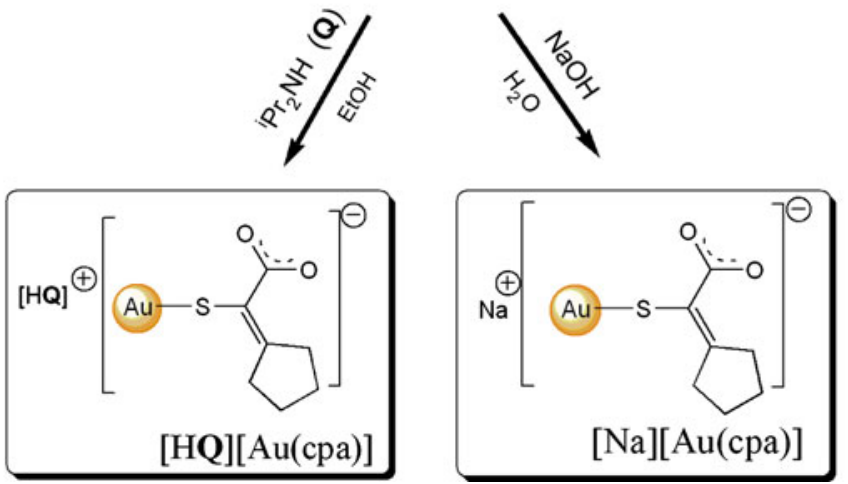

Yield: $75 \%$; mp: $190^{\circ} \mathrm{C}$. (Found: C 28.5, H 1.7, S 8.7\%. Calc. for $\mathrm{C}_{9} \mathrm{H}_{7} \mathrm{O}_{2} \mathrm{SAu}$ : C 28.7, H 1.9, S 8.5\%). IR $\left(\mathrm{cm}^{-1}\right): 1,680 \mathrm{vs}, \gamma(\mathrm{C}=\mathrm{O}) ; 1,446 \mathrm{~s}, \delta(\mathrm{OH}) ; 1,253 \mathrm{vs}$, $v(\mathrm{C}-\mathrm{O})$. NMR (DMSO-d $\left.\mathrm{d}_{6}\right):{ }^{1} \mathrm{H}, \delta 13.14$ (s, $1 \mathrm{H}, \mathrm{C}(1)$ $\mathrm{OH}), 7.84$ (s, 1H, C(3)H), 7.67 (d, 2H, C(5)H, C(9)H),
7.41 (pst, 2H, C(6)H, C(8)H), 7.28 (m, 1H, C(7)H); ${ }^{13} \mathrm{C}, \delta 166.0 \mathrm{C}(1), 128.8 \mathrm{C}(2), 144.3 \mathrm{C}(3), 133.7 \mathrm{C}(4)$, $130.6 \mathrm{C}(5)$ and $C(9), 128.3 \mathrm{C}(6)$ and $\mathrm{C}(8), 129.9 \mathrm{C}(7)$. [Au(Hfspa)] (2). $\mathrm{H}_{2}$ fspa $(0.06 \mathrm{~g}, 0.38 \mathrm{mmol})$, $\mathrm{NaAuCl}_{4} \cdot 3 \mathrm{H}_{2} \mathrm{O}(0.15 \mathrm{~g}, 0.38 \mathrm{mmol})$, thiodiglycol 
$\left(0.11 \mathrm{~cm}^{3}\right)$, ethanol $\left(3 \mathrm{~cm}^{3}\right), \mathrm{H}_{2} \mathrm{O}\left(5 \mathrm{~cm}^{3}\right)$, brown solid. Yield: $86 \%$; mp: $182^{\circ} \mathrm{C}$. (Found: C 22.8, H 1.4, S 8.5\%. Calc. for $\mathrm{C}_{7} \mathrm{H}_{5} \mathrm{O}_{3} \mathrm{SAu}$ : C 23.0, H 1.4, S 8.8\%). IR $\left(\mathrm{cm}^{-1}\right): 1,663 \mathrm{vs}, v(\mathrm{C}=\mathrm{O}) ; 1,466 \mathrm{vs}, \delta(\mathrm{OH}) ; 1,281$ vs br, $v\left(\mathrm{C}-\mathrm{O}\right.$ ). NMR (DMSO-d ${ }_{6}$ ): ${ }^{1} \mathrm{H}, \delta 12.97$ (brs, $1 \mathrm{H}, \mathrm{C}$ (1)OH), $7.61(\mathrm{~s}, 1 \mathrm{H}, \mathrm{C}(3) \mathrm{H}), 7.25(\mathrm{~d}, 1 \mathrm{H}, \mathrm{C}(5) \mathrm{H}), 6.68$ $(\mathrm{m}, 1 \mathrm{H}, \mathrm{C}(6) \mathrm{H}), 7.89(\mathrm{~d}, 1 \mathrm{H}, \mathrm{C}(7) \mathrm{H}) ;{ }^{13} \mathrm{C}, \delta 165.9 \mathrm{C}(1)$, $124.0 \mathrm{C}(2), 131.5 \mathrm{C}(3), 149.4 \mathrm{C}(4), 118.4 \mathrm{C}(5), 113.0$ $\mathrm{C}(6), 146.3 \mathrm{C}(7)$.

[Au(Htspa)] (3). $\mathrm{H}_{2}$ tspa $(0.07 \mathrm{~g}, 0.38 \mathrm{mmol})$, $\mathrm{NaAuCl}_{4} \cdot 3 \mathrm{H}_{2} \mathrm{O}(0.15 \mathrm{~g}, 0.38 \mathrm{mmol})$, thiodiglycol $\left(0.11 \mathrm{~cm}^{3}\right)$, ethanol $\left(3 \mathrm{~cm}^{3}\right), \mathrm{H}_{2} \mathrm{O}\left(5 \mathrm{~cm}^{3}\right)$, green solid. Yield: $85 \%$; mp: $207^{\circ} \mathrm{C}$. (Found: C 22.3, H 1.2, S $16.5 \%$. Calc. for $\mathrm{C}_{7} \mathrm{H}_{5} \mathrm{O}_{2} \mathrm{~S}_{2} \mathrm{Au}: \mathrm{C} 22.0, \mathrm{H} 1.3, \mathrm{~S}$ 16.8\%). IR $\left(\mathrm{cm}^{-1}\right)$ : $1,674 \mathrm{vs}, \vee(\mathrm{C}=\mathrm{O}) ; 1,408 \mathrm{~s}, \delta(\mathrm{OH})$; 1,270 vs, $v(\mathrm{C}-\mathrm{O})$. NMR (DMSO-d $\left.\mathrm{d}_{6}\right):{ }^{1} \mathrm{H}, \delta 12.57$ (brs, 1H, C(1)OH), 8.19 (s, 1H, C(3)H), $7.67(\mathrm{~d}, 1 \mathrm{H}, \mathrm{C}(5) \mathrm{H})$, 7.17 (pst, $1 \mathrm{H}, \mathrm{C}(6) \mathrm{H}), 7.89(\mathrm{~d}, 1 \mathrm{H}, \mathrm{C}(7) \mathrm{H}) ;{ }^{13} \mathrm{C}, \delta$ $166.1 \mathrm{C}(1), 123.0 \mathrm{C}(2), 137.6 \mathrm{C}(3), 137.6 \mathrm{C}(4), 140.3$ $\mathrm{C}(5), 127.1 \mathrm{C}(6), 134.2 \mathrm{C}(7)$.

[Au(H-o-pyspa)] (4). $\mathrm{H}_{2}$-o-pyspa (0.07 g, $\left.0.38 \mathrm{mmol}\right)$, $\mathrm{NaAuCl}_{4} \cdot 3 \mathrm{H}_{2} \mathrm{O}(0.15 \mathrm{~g}, 0.38 \mathrm{mmol})$, thiodiglycol $\left(0.11 \mathrm{~cm}^{3}\right)$, ethanol $\left(3 \mathrm{~cm}^{3}\right), \mathrm{H}_{2} \mathrm{O}\left(5 \mathrm{~cm}^{3}\right)$, pale yellow solid. Yield: $85 \%$; $\mathrm{mp}: 190^{\circ} \mathrm{C}$. (Found: C 25.4, H 1.5, N 3.4, S 8.3\%. Calc. for $\mathrm{C}_{8} \mathrm{H}_{6} \mathrm{O}_{2} \mathrm{SNAu}: \mathrm{C} 25.5, \mathrm{H} \mathrm{1.6}, \mathrm{N}$ 3.7, S $8.5 \%)$. IR $\left(\mathrm{cm}^{-1}\right): 1,694 \mathrm{~s}, v(\mathrm{C}=\mathrm{O}) ; 1,466 \mathrm{~m} \mathrm{br}$, $\delta(\mathrm{OH}) ; 1,250 \mathrm{vs}, \mathrm{v}(\mathrm{C}-\mathrm{O})$. NMR (DMSO-d $\left.\mathrm{d}_{6}\right):{ }^{1} \mathrm{H}, \delta 7.00$ (s, 1H, C(3)H), 8.30 (d, 1H, C(5)H), 8.10 (pst t $1 \mathrm{H}, \mathrm{C}(6)$ H), 7.22 (pst, $1 \mathrm{H}, \mathrm{C}(7) \mathrm{H}), 8.55(\mathrm{~d}, 1 \mathrm{H}, \mathrm{C}(8) \mathrm{H}) ;{ }^{13} \mathrm{C}, \delta$ 165.1 C(1), 136.0 C(2), 134.1 C(3), 154.5 C(4), 150.0 C (5), $134.0 \mathrm{C}(6), 121.0 \mathrm{C}(7), 124.0 \mathrm{C}(8)$.

[Au(HClpspa)] (5). $\mathrm{H}_{2} \mathrm{Clpspa}(0.08 \mathrm{~g}, 0.38 \mathrm{mmol})$, $\mathrm{NaAuCl}_{4} \cdot 3 \mathrm{H}_{2} \mathrm{O}(0.15 \mathrm{~g}, 0.38 \mathrm{mmol})$, thiodiglycol $\left(0.11 \mathrm{~cm}^{3}\right)$, ethanol $\left(3 \mathrm{~cm}^{3}\right), \mathrm{H}_{2} \mathrm{O}\left(5 \mathrm{~cm}^{3}\right)$, yellow solid. Yield: $84 \%$; mp: $183^{\circ} \mathrm{C}$. (Found: C 26.6, H 1.3, S 7.5\%. Calc. for $\mathrm{C}_{9} \mathrm{H}_{6} \mathrm{O}_{2} \mathrm{SClAu}$ : C 26.3, H 1.5, S 7.8\%). IR $\left(\mathrm{cm}^{-1}\right): 1,689 \mathrm{vs}, v(\mathrm{C}=\mathrm{O}) ; 1,436 \mathrm{~s}, \delta(\mathrm{OH}) ; 1,247 \mathrm{~s}$, $v(\mathrm{C}-\mathrm{O})$. NMR (DMSO- $\mathrm{d}_{6}$ ): ${ }^{1} \mathrm{H}, \delta 13.53$ (brs, $1 \mathrm{H}, \mathrm{C}$ (1)OH), 7.86 (s, 1H, C(3)H), 7.46 (d, 1H, C(6)H), 7.31 (pst t, 2H, C(7)H), 7.38 (pst, 2H, C(8)H), 7.61 (d, 1H, C (9)H); ${ }^{13} \mathrm{C}, \delta 166.6 \mathrm{C}(1), 127.8 \mathrm{C}(2), 139.7 \mathrm{C}(3), 132.5$ $\mathrm{C}(4), 133.5 \mathrm{C}(5), 130.7 \mathrm{C}(6), 131.3 \mathrm{C}(7), 126.9 \mathrm{C}(8)$, $129.3 \mathrm{C}(9)$.

[Au(H-o-mpspa)] (6). $\mathrm{H}_{2}$-o-mpspa (0.08 g, $\left.0.38 \mathrm{mmol}\right)$, $\mathrm{NaAuCl}_{4} \cdot 3 \mathrm{H}_{2} \mathrm{O}(0.15 \mathrm{~g}, 0.38 \mathrm{mmol})$, thiodiglycol $\left(0.11 \mathrm{~cm}^{3}\right)$, ethanol $\left(3 \mathrm{~cm}^{3}\right), \mathrm{H}_{2} \mathrm{O}\left(5 \mathrm{~cm}^{3}\right)$, pale yellow solid. Yield: $77 \%$; mp: $203^{\circ} \mathrm{C}$. (Found: C 29.2, H 2.5, S 8.0\%. Calc. for $\mathrm{C}_{10} \mathrm{H}_{9} \mathrm{O}_{3} \mathrm{SAu}$ : C 29.6, H 2.2, S 7.9\%). IR $\left(\mathrm{cm}^{-1}\right): 1,685 \mathrm{vs}, v(\mathrm{C}=\mathrm{O}) ; 1,463 \mathrm{~s}, \delta(\mathrm{OH}) ; 1,249 \mathrm{vs}$, $v(\mathrm{C}-\mathrm{O})$. NMR (DMSO-d $\left.{ }_{6}\right):{ }^{1} \mathrm{H}, \delta 13.15$ (brs, $1 \mathrm{H}, \mathrm{C}(1)$ $\mathrm{OH}), 8.00(\mathrm{~s}, 1 \mathrm{H}, \mathrm{C}(3) \mathrm{H}), 7.70(\mathrm{~d}, 1 \mathrm{H}, \mathrm{C}(6) \mathrm{H}), 6.97$ (pst, 1H, C(7)H), 7.40 (t, 1H, C(8)H), 7.05 (d, 1H, C(9) $\mathrm{H}), 3.79\left(\mathrm{~s}, 3 \mathrm{H}, \mathrm{OCH}_{3}\right) ;{ }^{13} \mathrm{C}, \delta 167.1 \mathrm{C}(1), 128.8 \mathrm{C}(2)$,
139.4 C(3), $122.3 \mathrm{C}(4), 157.5 \mathrm{C}(5), 111.1 \mathrm{C}(6), 131.6$ $\left.\mathrm{C}(7), 119.8 \mathrm{C}(8), 130.3 \mathrm{C}(9), 55.5 \mathrm{C}_{(\mathrm{OCH}}\right)$.

[Au(H-p-mpspa)] (7). $\mathrm{H}_{2}$-p-mpspa (0.08 g, $\left.0.38 \mathrm{mmol}\right)$, $\mathrm{NaAuCl}_{4} \cdot 3 \mathrm{H}_{2} \mathrm{O}(0.15 \mathrm{~g}, 0.38 \mathrm{mmol})$, thiodiglycol $\left(0.11 \mathrm{~cm}^{3}\right)$, ethanol $\left(3 \mathrm{~cm}^{3}\right), \mathrm{H}_{2} \mathrm{O}\left(5 \mathrm{~cm}^{3}\right)$, yellow solid. Yield: $92 \%$; mp: $215^{\circ} \mathrm{C}$. (Found: C 29.5, H 2.2, S 7.5\%. Calc. for $\mathrm{C}_{10} \mathrm{H}_{9} \mathrm{O}_{3} \mathrm{SAu}$ : $\mathrm{C} 29.6, \mathrm{H} 2.2, \mathrm{~S} 7.9 \%$ ). IR $\left(\mathrm{cm}^{-1}\right): 1,674 \mathrm{vs}, v(\mathrm{C}=\mathrm{O}) ; 1,438 \mathrm{~m}, \delta(\mathrm{OH}) ; 1,256 \mathrm{vs}$ br, $v(\mathrm{C}-\mathrm{O})$. NMR (DMSO-d $\left.{ }_{6}\right):{ }^{1} \mathrm{H}, \delta 13.10$ (brs, $1 \mathrm{H}, \mathrm{C}$ (1) $\mathrm{OH}), 7.79$ (s, 1H, C(3)H), 7.83 (d, 2H, C(5)H, C(9) $\mathrm{H}), 6.95(\mathrm{~d}, 2 \mathrm{H}, \mathrm{C}(6) \mathrm{H}, \mathrm{C}(8) \mathrm{H}), 3.80\left(\mathrm{~s}, 3 \mathrm{H}, \mathrm{OCH}_{3}\right)$; ${ }^{13} \mathrm{C}, \delta 166.5 \mathrm{C}(1), 125.1 \mathrm{C}(2), 144.8 \mathrm{C}(3), 126.2 \mathrm{C}(4)$, 133.0 $\mathrm{C}(5)$ and $\mathrm{C}(9), 113.8 \mathrm{C}(6)$ and $\mathrm{C}(8), 160.7 \mathrm{C}(7)$, $55.3 \mathrm{C}\left(\mathrm{OCH}_{3}\right)$.

[Au(H-o-hpspa)] (8). $\mathrm{H}_{2}$-o-hpspa (0.07 g, $\left.0.38 \mathrm{mmol}\right)$, $\mathrm{AuCl}_{4} \mathrm{Na} \cdot 3 \mathrm{H}_{2} \mathrm{O}(0.15 \mathrm{~g}, 0.38 \mathrm{mmol})$, thiodiglycol $\left(0.11 \mathrm{~cm}^{3}\right)$, ethanol $\left(3 \mathrm{~cm}^{3}\right), \mathrm{H}_{2} \mathrm{O}\left(5 \mathrm{~cm}^{3}\right)$, orange solid. Yield: $91 \%$; mp: $190^{\circ} \mathrm{C}$. (Found: C 27.6, H $1.8, \mathrm{~S} 8.6 \%$. Calc. for $\mathrm{C}_{9} \mathrm{H}_{7} \mathrm{O}_{3} \mathrm{SAu}$ : C 27.6, $\mathrm{H} 1.8$, S $8.2 \%)$. IR $\left(\mathrm{cm}^{-1}\right) 1,686$ vs, $v(\mathrm{C}=\mathrm{O}) ; 1,452 \mathrm{~s}$, $\delta(\mathrm{OH}) ; 1,250$ vs, $v(\mathrm{C}-\mathrm{O})$. NMR $\left(\mathrm{DMSO}^{\left.-\mathrm{d}_{6}\right)}{ }^{1} \mathrm{H}\right.$, $\delta 13.16$ (brs, $1 \mathrm{H}, \mathrm{C}(1) \mathrm{OH}), 7.97(\mathrm{~s}, 1 \mathrm{H}, \mathrm{C}(3) \mathrm{H})$, $9.99(\mathrm{~s}, 1 \mathrm{H}, \mathrm{C}(5) \mathrm{OH}), 7.84(\mathrm{~d}, 1 \mathrm{H}, \mathrm{C}(6) \mathrm{H}), 7.10$ (pst, 1H, C(7)H), 6.82 (pst, 1H, C(8)H), 7.75 (d, $1 \mathrm{H}, \mathrm{C}(9) \mathrm{H}) ;{ }^{13} \mathrm{C}, \delta 167.5 \mathrm{C}(1), 121.4 \mathrm{C}(2), 139.0$ $\mathrm{C}(3), 123.2 \mathrm{C}(4), 156.0 \mathrm{C}(5), 115.4 \mathrm{C}(6), 131.1 \mathrm{C}(7)$, $118.2 \mathrm{C}(8), 130.9 \mathrm{C}(9)$.

[Au(H-p-hpspa)] (9). $\mathrm{H}_{2}$-p-hpspa (0.07 g, $\left.0.38 \mathrm{mmol}\right)$, $\mathrm{NaAuCl}_{4} \cdot 3 \mathrm{H}_{2} \mathrm{O}(0.15 \mathrm{~g}, 0.38 \mathrm{mmol})$, thiodiglycol $\left(0.11 \mathrm{~cm}^{3}\right)$, ethanol $\left(3 \mathrm{~cm}^{3}\right), \mathrm{H}_{2} \mathrm{O}\left(5 \mathrm{~cm}^{3}\right)$, orange solid. Yield: $65 \%$; mp: $205^{\circ} \mathrm{C}$. (Found: C 27.5, H 2.0, S 8.2\%. Calc. for $\mathrm{C}_{9} \mathrm{H}_{7} \mathrm{O}_{3} \mathrm{SAu}$ : C 27.6, H 1.8, S 8.2\%). IR $\left(\mathrm{cm}^{-1}\right): 1,675$ vs, $v(\mathrm{C}=\mathrm{O}) ; 1,433 \mathrm{~m}, \delta(\mathrm{OH}) ; 1,245 \mathrm{~s}$ br, $v(\mathrm{C}-\mathrm{O})$. NMR (DMSO-d $\left.{ }_{6}\right):{ }^{1} \mathrm{H}, \delta 12.91$ (brs, $1 \mathrm{H}, \mathrm{C}$ (1) $\mathrm{OH}), 7.74$ (s, 1H, C(3)H), 7.77 (d, 2H, C(5)H, C(9) H), 6.77 (d, 2H, C(6)H, C(8)H), 10.18 (s, 1H, C(7)OH); ${ }^{13} \mathrm{C}, \delta 166.7 \mathrm{C}(1), 123.6 \mathrm{C}(2), 145.4 \mathrm{C}(3), 124.7 \mathrm{C}(4)$, $133.4 \mathrm{C}(5)$ and $\mathrm{C}(9), 115.3 \mathrm{C}(6)$ and $\mathrm{C}(8), 159.7 \mathrm{C}(7)$. [Au(H-diBr-o-hpspa)] (10). $\mathrm{H}_{2}$ diBr-o-hpspa $(0.13 \mathrm{~g}$, $0.38 \mathrm{mmol}), \mathrm{NaAuCl}_{4} \cdot 3 \mathrm{H}_{2} \mathrm{O}(0.15 \mathrm{~g}, 0.38 \mathrm{mmol})$, thiodiglycol $\left(0.11 \mathrm{~cm}^{3}\right)$, ethanol $\left(3 \mathrm{~cm}^{3}\right), \mathrm{H}_{2} \mathrm{O}\left(5 \mathrm{~cm}^{3}\right)$, yellow solid. Yield: $83 \%$; mp: $238^{\circ} \mathrm{C}$. (Found: $\mathrm{C} 19.8$, $\mathrm{H} 0.8$, S 5.5\%. Calc. for $\mathrm{C}_{9} \mathrm{H}_{5} \mathrm{O}_{3} \mathrm{SBr}_{2} \mathrm{Au}$ : C 19.6, $\mathrm{H} 0.9$, S $5.8 \%)$. IR $\left(\mathrm{cm}^{-1}\right): 1,690$ vs, $v(\mathrm{C}=\mathrm{O}) ; 1,448 \mathrm{vs,}$ $\delta(\mathrm{OH}) ; 1,255$ vs, $v(\mathrm{C}-\mathrm{O})$. NMR $\left(\mathrm{DMSO}^{\left.-\mathrm{d}_{6}\right)}:{ }^{1} \mathrm{H}, \delta\right.$ 13.30 (brs, $1 \mathrm{H}, \mathrm{C}(1) \mathrm{OH}), 7.87$ (s, $1 \mathrm{H}, \mathrm{C}(3) \mathrm{H}), 9.95$ (brs, 1H, C(5)OH), $7.75(\mathrm{~s}, 1 \mathrm{H}, \mathrm{C}(7) \mathrm{H}), 7.59(\mathrm{~s}, 1 \mathrm{H}$, $\mathrm{C}(9) \mathrm{H}) ;{ }^{13} \mathrm{C}, \delta 166.7 \mathrm{C}(1), 126.5 \mathrm{C}(2), 132.1 \mathrm{C}(3)$, $127.0 \mathrm{C}(4), 151.3 \mathrm{C}(5), 112.8 \mathrm{C}(6), 137.4 \mathrm{C}(7), 110.6$ $\mathrm{C}(8), 135.1 \mathrm{C}(9)$.

[Au(Hcpa)] (11). $\mathrm{H}_{2}$ cpa $(0.06 \mathrm{~g}, 0.38 \mathrm{mmol})$, $\mathrm{NaAuCl}_{4} \cdot 3 \mathrm{H}_{2} \mathrm{O}(0.15 \mathrm{~g}, 0.38 \mathrm{mmol})$, thiodiglycol $\left(0.11 \mathrm{~cm}^{3}\right)$, ethanol $\left(3 \mathrm{~cm}^{3}\right), \mathrm{H}_{2} \mathrm{O}\left(5 \mathrm{~cm}^{3}\right)$, brown solid. 
Yield: $80 \%$; mp: $207^{\circ} \mathrm{C}$. (Found: C 23.5, H 2.6, S 9.3\%. Calc. for $\left.\mathrm{C}_{7} \mathrm{H}_{9} \mathrm{O}_{2} \mathrm{SAu}: \mathrm{C} 23.7, \mathrm{H} 2.6, \mathrm{~S} 9.0 \%\right)$. IR $\left(\mathrm{cm}^{-1}\right)$ : 1,668 vs, $v(\mathrm{C}=\mathrm{O}) ; 1,413 \mathrm{vs}, \delta(\mathrm{OH}) ; 1,274 \mathrm{vs}$, $v(\mathrm{C}-\mathrm{O})$. NMR (DMSO-d $\left.{ }_{6}\right):{ }^{1} \mathrm{H}, \delta 12.52$ (brs, $1 \mathrm{H}, \mathrm{C}(1)$ $\mathrm{OH}), 2.67$ (m, 2H, C(4) $\left.\mathrm{H}_{2}\right), 1.67\left(\mathrm{~m}, 2 \mathrm{H}, \mathrm{C}(5) \mathrm{H}_{2}\right), 1.57$ $\left(\mathrm{m}, 2 \mathrm{H}, \mathrm{C}(6) \mathrm{H}_{2}\right), 2.56\left(\mathrm{~m}, 2 \mathrm{H}, \mathrm{C}(7) \mathrm{H}_{2}\right) ;{ }^{13} \mathrm{C}, \delta 171.3 \mathrm{C}$ (1), 119.7 C(2), 166.5 C(3), 36.0 C(4), $26.5 \mathrm{C}(5), 25.0$ $\mathrm{C}(6), 35.0 \mathrm{C}(7)$.

Complexes of the type $[H Q][A u(L)]$ Complexes 12-22 $(\mathrm{HQ}=$ diisopropylammonium) were prepared by adding diisopropylamine to a suspension of the appropriate [Au(HL)] complex in ethanol. The mixture was stirred at room temperature for $24 \mathrm{~h}$. The resulting solid was filtered off and dried in vacuo, and the ethanol was evaporated from the filtrate at room temperature.

[HQ][Au(pspa)] (12). [Au(Hpspa)] (0.08 g, $0.20 \mathrm{mmol})$, diisopropylamine $\left(0.03 \mathrm{~cm}^{3} 0.20 \mathrm{mmol}\right)$, ethanol $\left(9 \mathrm{~cm}^{3}\right)$, white solid. Yield: $75 \%$; mp: $207^{\circ} \mathrm{C}$. (Found: C 37.5, H 4.2, S 6.5, N 2.8\%. Calc. for $\mathrm{C}_{15} \mathrm{H}_{22} \mathrm{O}_{2} \mathrm{SNAu}$ : C $37.7, \mathrm{H} 4.6, \mathrm{~S} 6.7, \mathrm{~N} \mathrm{2.9 \% ).} \mathrm{IR}\left(\mathrm{cm}^{-1}\right): 1,629 \mathrm{~s}$, $v\left(\mathrm{NH}_{2}{ }^{+}\right) ; 1,569 \mathrm{~s}, \nu_{\text {asym }}\left(\mathrm{CO}_{2}{ }^{-}\right) ; 1,345 \mathrm{vs}, \nu_{\text {sym }}\left(\mathrm{CO}_{2}{ }^{-}\right)$. NMR (DMSO-d $\left.)_{6}\right):{ }^{1} \mathrm{H}, \delta 7.60(\mathrm{~s}, 1 \mathrm{H}, \mathrm{C}(3) \mathrm{H}), 7.42(\mathrm{~d}$, 2H, C(5)H, C(9)H), 7.33 (pst, 2H, C(6)H, C(8)H), 7.20 $(\mathrm{m}, 1 \mathrm{H}, \mathrm{C}(7) \mathrm{H}), 1.19$ (d, 12H, [Q]CH $\mathrm{CH}_{3}, 3.23(\mathrm{~m}, 2 \mathrm{H},[\mathrm{Q}]$ $\mathrm{CH}) ;{ }^{13} \mathrm{C}, \delta 171.9 \mathrm{C}(1), 127.4 \mathrm{C}(2), 143.0 \mathrm{C}(3), 136.8 \mathrm{C}$ (4), $130.3 \mathrm{C}(5)$ and $\mathrm{C}(9), 128.2 \mathrm{C}(6)$ and $\mathrm{C}(8), 129.6 \mathrm{C}$ (7), $46.1 \mathrm{CH}[\mathrm{HQ}], 19.6 \mathrm{CH}_{3}[\mathrm{HQ}]$.

[HQ][Au(fspa)] (13). [Au(Hfspa)] (0.07 g, $0.20 \mathrm{mmol})$, diisopropylamine $\left(0.03 \mathrm{~cm}^{3} 0.20 \mathrm{mmol}\right)$, ethanol $\left(8 \mathrm{~cm}^{3}\right)$, brown solid. Yield: $63 \%$; mp: $194^{\circ} \mathrm{C}$. (Found: C 33.2, H 4.3, S 6.9, N 2.8\%. Calc. for $\mathrm{C}_{13} \mathrm{H}_{20} \mathrm{O}_{3} \mathrm{SNAu}$ : C 33.4, H 4.3, S 6.9, N 3.0\%). IR $\left(\mathrm{cm}^{-1}\right)$ : 1,621 s br, $v\left(\mathrm{NH}_{2}{ }^{+}\right) ; 1,553 \mathrm{~s} \mathrm{br}, v_{\text {asym }}\left(\mathrm{CO}_{2}{ }^{-}\right) ; 1,339 \mathrm{vs}, v_{\text {sym }}\left(\mathrm{CO}_{2}{ }^{-}\right)$. NMR (DMSO-d $\left.)_{6}\right){ }^{1} \mathrm{H}, \delta 7.40(\mathrm{~s}, 1 \mathrm{H}, \mathrm{C}(3) \mathrm{H}), 7.13$ (d, 1H, C(5)H), 6.55 (m, 1H, C(6)H), 7.66 (d, 1H, C(7)H), 1.24 (d, 12H, [Q]CH 3$), 3.29$ (m, 2H, [Q]CH), 8.97 (s, 2H, $\left.[\mathrm{Q}] \mathrm{NH}_{2}{ }^{+}\right) ;{ }^{13} \mathrm{C}, \delta 170.2 \mathrm{C}(1), 34.8 \mathrm{C}(2), 122.8 \mathrm{C}(3)$, $152.6 \mathrm{C}(4), 111.8 \mathrm{C}(5), 110.5 \mathrm{C}(6), 141.8 \mathrm{C}(7), 5.3 \mathrm{CH}$ [HQ], $19.3 \mathrm{CH}_{3}$ [HQ].

[HQ][Au(tspa)] (14). [Au(Htspa)] (0.07 g, $0.16 \mathrm{mmol})$, diisopropylamine $\left(0.024 \mathrm{~cm}^{3} 0.16 \mathrm{mmol}\right)$, ethanol $\left(8 \mathrm{~cm}^{3}\right)$, brown solid. Yield: $53 \%$; mp: $203^{\circ} \mathrm{C}$. (Found: C 32.0, H 4.3, S 13.4, N 2.6\%. Calc. for $\mathrm{C}_{13} \mathrm{H}_{24} \mathrm{O}_{2} \mathrm{~S}_{2-}$ NAu: C 32.3, H 4.2, S 13.3, N 2.9\%). IR ( $\left.\mathrm{cm}^{-1}\right): 1,622$ vs, $v\left(\mathrm{NH}_{2}{ }^{+}\right) ; 1,565 \mathrm{vs}, v_{\text {asym }}\left(\mathrm{CO}_{2}{ }^{-}\right) ; 1,334 \mathrm{vs}$, $v_{\text {sym }}\left(\mathrm{CO}_{2}{ }^{-}\right)$. NMR (DMSO-d $):{ }^{1} \mathrm{H}, \delta 7.77(\mathrm{~s}, 1 \mathrm{H}, \mathrm{C}(3)$ $\mathrm{H}), 7.31$ (d, 1H, C(5)H), 7.04 (pst, 1H, C(6)H), 7.54 (d, 1H, C(7)H), 1.24 (d, 12H, [Q]CH $), 3.27$ (m, 2H, [Q] $\mathrm{CH}), 9.07$ (s, 2H, [Q] $\left.\mathrm{NH}_{2}{ }^{+}\right) ;{ }^{13} \mathrm{C}, \delta 169.9 \mathrm{C}(1), 24.9 \mathrm{C}(2)$, 36.1 C(3), $41.9 \mathrm{C}(4), 132.5 \mathrm{C}(5), 126.7 \mathrm{C}(6), 126.2 \mathrm{C}(7)$, 5.6 CH[HQ], $18.9 \mathrm{CH}_{3}[\mathrm{HQ}]$.
[HQ][Au(-o-pyspa)] (15). [Au(H-o-pyspa)] (0.06 g, $0.17 \mathrm{mmol})$, diisopropylamine $\left(0.026 \mathrm{~cm}^{3}, 0.17 \mathrm{mmol}\right)$, ethanol $\left(7 \mathrm{~cm}^{3}\right)$, orange solid. Yield: $76 \%$; mp: $194^{\circ} \mathrm{C}$. (Found: $\mathrm{C}$ 34.9, $\mathrm{H}$ 4.6, S 6.5, N 5.6\%. Calc. for $\mathrm{C}_{14} \mathrm{H}_{21} \mathrm{O}_{2} \mathrm{SN}_{2} \mathrm{Au}: \mathrm{C} 35.1, \mathrm{H} 4.4, \mathrm{~S} 6.7, \mathrm{~N} 5.9 \%$ ). IR $\left(\mathrm{cm}^{-1}\right): 1,610 \mathrm{~m} \mathrm{sh}, v\left(\mathrm{NH}_{2}{ }^{+}\right) ; 1,579 \mathrm{vs}, \mathrm{v}_{\text {asym }}\left(\mathrm{CO}_{2}{ }^{-}\right)$; $1,354 \mathrm{vs}, \mathrm{v}_{\mathrm{sym}}\left(\mathrm{CO}_{2}{ }^{-}\right)$. NMR (DMSO-d $\left.{ }_{6}\right):{ }^{1} \mathrm{H}, \delta 6.99(\mathrm{~s}$, 1H, C(3)H), 7.63 (d, 1H, C(5)H), 7.82 (pst, 1H, C(6)H), 7.20 (pst, 1H, C(7)H), 8.32 (d, 1H, C (8)H), 3.18 (d, $\left.12 \mathrm{H},[\mathrm{Q}] \mathrm{CH}_{3}\right), 1.19(\mathrm{~m}, 2 \mathrm{H},[\mathrm{Q}] \mathrm{CH}) ;{ }^{13} \mathrm{C}, \delta 170.3 \mathrm{C}(1)$, $134.8 \mathrm{C}(2), 137.2 \mathrm{C}(3), 152.0 \mathrm{C}(4), 143.5 \mathrm{C}(5), 130.3$ $\mathrm{C}(6), 122.3 \mathrm{C}(7), 126.8 \mathrm{C}(8), 45.9 \mathrm{CH}[\mathrm{HQ}], 18.8$ $\mathrm{CH}_{3}[\mathrm{HQ}]$.

[HQ][Au(Clpspa)] (16). [Au(HClpspa)] (0.05 g, $0.16 \mathrm{mmol})$, diisopropylamine $\left(0.024 \mathrm{~cm}^{3} 0.16 \mathrm{mmol}\right)$, ethanol $\left(6 \mathrm{~cm}^{3}\right)$, white solid. Yield: $88 \%$; mp: $179^{\circ} \mathrm{C}$. (Found: $\mathrm{C}$ 34.9, H 4.2, S 6.4, N 2.8\%. Calc. for $\mathrm{C}_{15} \mathrm{H}_{21} \mathrm{O}_{2} \mathrm{SNClAu}$ : C 35.2, H 4.1, S 6.3, N 2.7\%). IR $\left(\mathrm{cm}^{-1}\right): 1,623 \mathrm{vs}, v\left(\mathrm{NH}_{2}{ }^{+}\right) ; 1,570 \mathrm{vs} \mathrm{br}, v_{\text {asym }}\left(\mathrm{CO}_{2}{ }^{-}\right)$; $1,346 \mathrm{vs}, \mathrm{v}_{\mathrm{sym}}\left(\mathrm{CO}_{2}{ }^{-}\right)$. NMR (DMSO-d $\left.{ }_{6}\right):{ }^{1} \mathrm{H}, \delta 7.66(\mathrm{~s}$, 1H, C(3)H), 7.40 (d, 1H, C(6)H), $7.13(\mathrm{~m}, 1 \mathrm{H}, \mathrm{C}(7) \mathrm{H}$, $\mathrm{C}(8) \mathrm{H}), 7.47(\mathrm{~d}, 1 \mathrm{H}, \mathrm{C}(9) \mathrm{H}), 1.21\left(\mathrm{~d}, 12 \mathrm{H},[\mathrm{Q}] \mathrm{CH}_{3}\right)$, $3.29(\mathrm{~m}, 2 \mathrm{H},[\mathrm{Q}] \mathrm{CH}), 9.10\left(\mathrm{~s}, 2 \mathrm{H},[\mathrm{Q}] \mathrm{NH}_{2}{ }^{+}\right) ;{ }^{13} \mathrm{C}, \delta$ $171.8 \mathrm{C}(1), 125.8 \mathrm{C}(2), 136.5 \mathrm{C}(3), 135.1 \mathrm{C}(4), 134.3$ $\mathrm{C}(5), 130.2 \mathrm{C}(6), 131.0 \mathrm{C}(7), 136.6 \mathrm{C}(8), 128.9 \mathrm{C}(9)$, $45.8 \mathrm{CH}[\mathrm{HQ}], 19.2 \mathrm{CH}_{3}[\mathrm{HQ}]$.

[HQ][Au(-o-mpspa)] (17). [Au(H-o-mpspa)] (0.07 g, $0.17 \mathrm{mmol})$, diisopropylamine $\left(0.027 \mathrm{~cm}^{3}, 0.17 \mathrm{mmol}\right)$, ethanol $\left(8 \mathrm{~cm}^{3}\right)$, beige solid. Yield: $66 \%$; mp: $177^{\circ} \mathrm{C}$. (Found: $\mathrm{C}$ 37.4, $\mathrm{H}$ 4.5, $\mathrm{S}$ 6.8, N 2.5\%. Calc. for $\mathrm{C}_{16} \mathrm{H}_{24} \mathrm{O}_{3} \mathrm{SNAu}: \mathrm{C} 37.9, \mathrm{H} 4.8, \mathrm{~S} 6.3, \mathrm{~N} 2.8 \%$ ). IR $\left(\mathrm{cm}^{-1}\right)$ : 1,620 vs, $v\left(\mathrm{NH}_{2}{ }^{+}\right) ; 1,565 \mathrm{vs}, \mathrm{v}_{\text {asym }}\left(\mathrm{CO}_{2}{ }^{-}\right)$; $1,346 \mathrm{vs}, \mathrm{v}_{\text {sym }}\left(\mathrm{CO}_{2}{ }^{-}\right)$. NMR (DMSO-d $\left.{ }_{6}\right):{ }^{1} \mathrm{H}, \delta 7.53$ (s, 1H, C(3)H), 7.67 (d, 1H, C(6)H), 6.90 (m, 2H, C(7) $\mathrm{H}$ and $\mathrm{C}(9) \mathrm{H}), 7.15$ (pst, $1 \mathrm{H}, \mathrm{C}(8) \mathrm{H}), 3.75$ (s, 3H, $\left.\mathrm{OCH}_{3}\right), 1.21\left(\mathrm{~d}, 12 \mathrm{H},[\mathrm{Q}] \mathrm{CH}_{3}\right), 3.24(\mathrm{~m}, 2 \mathrm{H},[\mathrm{Q}] \mathrm{CH})$, $8.80\left(\mathrm{~s}, 2 \mathrm{H},[\mathrm{Q}] \mathrm{NH}_{2}{ }^{+}\right) ;{ }^{13} \mathrm{C}, \delta 168.7 \mathrm{C}(1), 129.0 \mathrm{C}(2)$, $137.3 \mathrm{C}(3), 125.0 \mathrm{C}(4), 156.8 \mathrm{C}(5), 110.5 \mathrm{C}(6), 133.4$ $\mathrm{C}(7), 119.3 \mathrm{C}(8), 130.6 \mathrm{C}(9), 55.1 \mathrm{C}\left(\mathrm{OCH}_{3}\right), 45.5 \mathrm{CH}$ [HQ], $19.0 \mathrm{CH}_{3}$ [HQ].

[HQ][Au(-p-mpspa)] (18). [Au(H-p-mpspa)] (0.07 g, $0.17 \mathrm{mmol})$, diisopropylamine $\left(0.027 \mathrm{~cm}^{3}, 0.17 \mathrm{mmol}\right)$, ethanol $\left(8 \mathrm{~cm}^{3}\right)$, pale orange solid. Yield: $67 \%$; mp: $198^{\circ} \mathrm{C}$. (Found: C 37.9, H 4.6, S 6.2, N 2.6\%. Calc. for $\mathrm{C}_{16} \mathrm{H}_{24} \mathrm{O}_{3}$ SNAu: $\mathrm{C} 37.9, \mathrm{H} 4.8, \mathrm{~S} 6.3, \mathrm{~N} 2.8 \%$ ). IR $\left(\mathrm{cm}^{-1}\right): 1,627 \mathrm{~m}, v\left(\mathrm{NH}_{2}{ }^{+}\right) ; 1,570 \mathrm{vs}, v_{\mathrm{asym}}\left(\mathrm{CO}_{2}{ }^{-}\right)$; $1,348 \mathrm{vs}, \mathrm{v}_{\mathrm{sym}}\left(\mathrm{CO}_{2}{ }^{-}\right)$. NMR (DMSO-d $\left.{ }_{6}\right):{ }^{1} \mathrm{H}, \delta 7.41$ $(\mathrm{s}, 1 \mathrm{H}, \mathrm{C}(3) \mathrm{H}), 7.66(\mathrm{~d}, 2 \mathrm{H}, \mathrm{C}(5) \mathrm{H}, \mathrm{C}(9) \mathrm{H}), 6.88(\mathrm{~d}$, $2 \mathrm{H}, \mathrm{C}(6) \mathrm{H}, \mathrm{C}(8) \mathrm{H}), 3.70$ (s, 3H, $\left.\mathrm{OCH}_{3}\right), 1.22$ (d, 12H, $\left.[\mathrm{Q}] \mathrm{CH}_{3}\right), 3.24(\mathrm{~m}, 2 \mathrm{H},[\mathrm{Q}] \mathrm{CH}), 9.09$ (s, 2H, [Q]NH$\left.{ }_{2}^{+}\right)$; ${ }^{13} \mathrm{C}, \delta 169.0 \mathrm{C}(1), 114.2 \mathrm{C}(2), 134.9 \mathrm{C}(3), 131.1 \mathrm{C}(4)$, $131.4 \mathrm{C}(5)$ and $\mathrm{C}(9), 113.2 \mathrm{C}(6)$ and $\mathrm{C}(8), 158.5 \mathrm{C}(7)$, $54.9 \mathrm{C}\left(\mathrm{OCH}_{3}\right), 45.5 \mathrm{CH}[\mathrm{HQ}], 19.1 \mathrm{CH}_{3}[\mathrm{HQ}]$. 
[HQ][Au(-o-hpspa)] (19). [Au(H-o-hpspa)] (0.06 g, $0.17 \mathrm{mmol})$, diisopropylamine $\left(0.027 \mathrm{~cm}^{3}, 0.17 \mathrm{mmol}\right)$, ethanol $\left(7 \mathrm{~cm}^{3}\right)$, pale orange solid. Yield: $70 \%$; mp: $192^{\circ} \mathrm{C}$. (Found: C 36.3, H 4.2, S 6.6, N 2.6\%. Calc. for $\mathrm{C}_{15} \mathrm{H}_{22} \mathrm{O}_{3}$ SNAu: C 36.5, H 4.5, S 6.5, N 2.8\%). IR $\left(\mathrm{cm}^{-1}\right)$ : 1,600 vs, $v\left(\mathrm{NH}_{2}{ }^{+}\right)$; $1,558 \mathrm{vs}, v_{\text {asym }}\left(\mathrm{CO}_{2}^{-}\right)$; 1,350 vs, $v_{\text {sym }}\left(\mathrm{CO}_{2}{ }^{-}\right)$. NMR (DMSO-d $\left.\mathrm{d}_{6}\right):{ }^{1} \mathrm{H}, \delta 7.70$ (s, 1H, C(3)H), 8.52 (s, 1H, C(5)OH), $6.86(\mathrm{~d}, 1 \mathrm{H}, \mathrm{C}(6)$ H), 7.05 (pst, 1H, C(7)H), 6.68 (pst, $1 \mathrm{H}, \mathrm{C}(8) \mathrm{H}), 7.87$ $(\mathrm{d}, 1 \mathrm{H}, \mathrm{C}(9) \mathrm{H}), 3.21\left(\mathrm{~d}, 12 \mathrm{H},[\mathrm{Q}] \mathrm{CH}_{3}\right), 1.20(\mathrm{~m}, 2 \mathrm{H}$, $[\mathrm{Q}] \mathrm{CH}) ;{ }^{13} \mathrm{C}, \delta 171.3 \mathrm{C}(1), 122.3 \mathrm{C}(2), 136.6 \mathrm{C}(3)$, 128.2 C(4), $155.7 \mathrm{C}(5), 115.0 \mathrm{C}(6), 131.3 \mathrm{C}(7), 118.6$ $\mathrm{C}(8), 131.5 \mathrm{C}(9), 45.5 \mathrm{CH}[\mathrm{HQ}], 18.7 \mathrm{CH}_{3}[\mathrm{HQ}]$.

[HQ][Au(-p-hpspa)] (20). [Au(H-p-hpspa)] $(0.05 \mathrm{~g}$, $0.11 \mathrm{mmol})$, diisopropylamine $\left(0.016 \mathrm{~cm}^{3} 0.11 \mathrm{mmol}\right)$, ethanol $\left(6 \mathrm{~cm}^{3}\right)$, pale yellow solid. Yield: $82 \%$; $\mathrm{mp}$ : $207^{\circ} \mathrm{C}$. (Found: C 36.3, H 4.7, S 6.6, N 2.6\%. Calc. for $\mathrm{C}_{15} \mathrm{H}_{22} \mathrm{O}_{3}$ SNAu: C 36.5, H 4.5, S 6.5, N 2.8\%). IR $\left(\mathrm{cm}^{-1}\right)$ : $1,606 \mathrm{vs}, v\left(\mathrm{NH}_{2}^{+}\right) ; 1,558 \mathrm{vs}, v_{\text {asym }}\left(\mathrm{CO}_{2}{ }^{-}\right) ; 1,345$ vs, $v_{\text {sym }}\left(\mathrm{CO}_{2}{ }^{-}\right)$. NMR (DMSO-d $\left.{ }_{6}\right):{ }^{1} \mathrm{H}, \delta 7.57$ (s, $1 \mathrm{H}, \mathrm{C}$ (3)H), $7.82(\mathrm{~d}, 2 \mathrm{H}, \mathrm{C}(5) \mathrm{H}, \mathrm{C}(9) \mathrm{H}), 6.75(\mathrm{~d}, 2 \mathrm{H}, \mathrm{C}(6) \mathrm{H}, \mathrm{C}$ (8)H), 8.90 (s, 1H, C(7)OH), 3.27 (d, 12H, [Q]CH $), 1.19$ (m, 2H, [Q]CH), $9.60\left(\mathrm{~s}, 2 \mathrm{H},[\mathrm{Q}] \mathrm{NH}_{2}{ }^{+}\right) ;{ }^{13} \mathrm{C}, \delta 171.2 \mathrm{C}$ (1), 124.3 C(2), 129.0 C(4), 131.5 C(5) and C(9), 114.5 C (6) and $\mathrm{C}(8), 158.4 \mathrm{C}(7), 45.5 \mathrm{CH}[\mathrm{HQ}], 20.0 \mathrm{CH}_{3}[\mathrm{HQ}]$. [HQ][Au(-diBr-o-hpspa)] (21). [Au(H-diBr-o-hpspa)] $(0.07 \mathrm{~g}, 0.20 \mathrm{mmol})$, diisopropylamine $\left(0.03 \mathrm{~cm}^{3}\right.$ $0.20 \mathrm{mmol})$, ethanol $\left(8 \mathrm{~cm}^{3}\right)$, yellow solid. Yield: $76 \%$; mp: $189^{\circ} \mathrm{C}$. (Found: C 34.9, H 4.5, S 5.5, N 5.5\%. Calc. for $\mathrm{C}_{15} \mathrm{H}_{20} \mathrm{O}_{3} \mathrm{SBr}_{2} \mathrm{NAu}$ : C 27.7, H 3.1, S 4.9, N $2.1 \%)$. IR $\left(\mathrm{cm}^{-1}\right): 1,605 \mathrm{~s} \mathrm{br}, v\left(\mathrm{NH}_{2}^{+}\right) ; 1,566 \mathrm{vs} \mathrm{br}$, $v_{\text {asym }}\left(\mathrm{CO}_{2}{ }^{-}\right) ; 1,347$ vs, $v_{\text {sym }}\left(\mathrm{CO}_{2}{ }^{-}\right)$. NMR (DMSO-d $\left.{ }_{6}\right)$ : ${ }^{1} \mathrm{H}, \delta 7.67$ (s, $\left.1 \mathrm{H}, \mathrm{C}(3) \mathrm{H}\right), 0.80$ (br, $\left.1 \mathrm{H}, \mathrm{C}(5) \mathrm{OH}\right), 7.54$ (s, $1 \mathrm{H}, \mathrm{C}(7) \mathrm{H}), 7.32(\mathrm{~s}, 1 \mathrm{H}, \mathrm{C}(9) \mathrm{H}), 3.20\left(\mathrm{~d}, 12 \mathrm{H},[\mathrm{Q}] \mathrm{CH}_{3}\right)$, $1.13(\mathrm{~m}, 2 \mathrm{H},[\mathrm{Q}] \mathrm{CH}) ;{ }^{13} \mathrm{C}, \delta 171.6 \mathrm{C}(1), 126.0 \mathrm{C}(2), 135.6$ $\mathrm{C}(3), 126.8 \mathrm{C}(4), 155.3 \mathrm{C}(5), 116.4 \mathrm{C}(6), 136.3 \mathrm{C}(7)$, $113.0 \mathrm{C}(8), 132.8 \mathrm{C}(9), 45.8 \mathrm{CH}[\mathrm{HQ}], 19.8 \mathrm{CH}_{3}[\mathrm{HQ}]$.

[HQ][Au(cpa)] (22). [Au(Hcpa)] (0.07 g, 0.18 mmol), diisopropylamine $\left(0.028 \mathrm{~cm}^{3} 0.18 \mathrm{mmol}\right)$, ethanol $\left(8 \mathrm{~cm}^{3}\right)$, beige solid. Yield: $58 \%$; mp: $172^{\circ} \mathrm{C}$. (Found: $\mathrm{C}$ 34.3, H 5.1, $\mathrm{S} 6.8$, N 3.2\%. Calc. for $\mathrm{C}_{13} \mathrm{H}_{24} \mathrm{O}_{2} \mathrm{SNAu}: \mathrm{C}$ 34.3, H 5.3, S 7.0, N 3.1\%). IR $\left(\mathrm{cm}^{-1}\right)$ : 1,617 vs, $v\left(\mathrm{NH}_{2}{ }^{+}\right) ; 1,543$ vs, $v_{\text {asym }}\left(\mathrm{CO}_{2}{ }^{-}\right) ; 1,357 \mathrm{vs}, v_{\text {sym }}\left(\mathrm{CO}_{2}{ }^{-}\right)$. NMR (DMSO-d $\left.\mathrm{d}_{6}\right){ }^{1} \mathrm{H}, \delta 2.57\left(\mathrm{~m}, 2 \mathrm{H}, \mathrm{C}(4) \mathrm{H}_{2}\right), 1.65(\mathrm{~m}$, $\left.2 \mathrm{H}, \mathrm{C}(5) \mathrm{H}_{2}\right), 1.65\left(\mathrm{~m}, 2 \mathrm{H}, \mathrm{C}(6) \mathrm{H}_{2}\right), 2.57(\mathrm{~m}, 2 \mathrm{H}, \mathrm{C}(7)$ $\left.\mathrm{H}_{2}\right), 3.22\left(\mathrm{~d}, 12 \mathrm{H},[\mathrm{Q}] \mathrm{CH}_{3}\right), 1.13(\mathrm{~m}, 2 \mathrm{H},[\mathrm{Q}] \mathrm{CH}) ;{ }^{13} \mathrm{C}, \delta$ 171.5 C(1), 133.2 C(2), 61.3 C(3), 37.2 C(4), 28.0 C(5), $26.1 \mathrm{C}(6), 35.1 \mathrm{C}(7), 45.5 \mathrm{CH}[\mathrm{HQ}], 19.2 \mathrm{CH}_{3}[\mathrm{HQ}]$.

Complexes of the type $\mathrm{Na}[\mathrm{Au}(\mathrm{L})] \cdot \mathrm{H}_{2} \mathrm{O}$ Complexes 23-33 were prepared by adding $\mathrm{NaOH}$ to a suspension of the appropriate $[\mathrm{Au}(\mathrm{HL})]$ complex in water. The mixture was stirred at room temperature for $24 \mathrm{~h}$, the solution was passed through a folded filter paper (Whatman No. 42) and the solvent was evaporated at room temperature.

$\mathrm{Na}\left[\mathrm{Au}(\right.$ pspa) $] \cdot \mathrm{H}_{2} \mathrm{O}$ (23). [Au(Hpspa)] (0.05 g, $0.13 \mathrm{mmol}), \mathrm{NaOH}(0.005 \mathrm{~g}, 0.13 \mathrm{mmol}), \mathrm{H}_{2} \mathrm{O}\left(5 \mathrm{~cm}^{3}\right)$, pale yellow solid. Yield: $55 \%$; mp: $215^{\circ} \mathrm{C}$. (Found: C 26.0, H 1.8, S 7.2\%. Calc. for $\mathrm{C}_{9} \mathrm{H}_{8} \mathrm{O}_{3} \mathrm{SAuNa}$ : C 26.0, $\mathrm{H}$ $1.9, \mathrm{~S} 7.7 \%)$. IR $\left(\mathrm{cm}^{-1}\right): 1,573 \mathrm{vs}, v_{\text {asym }}\left(\mathrm{CO}_{2}{ }^{-}\right) ; 1,368 \mathrm{vs}$, $v_{\text {sym }}\left(\mathrm{CO}_{2}{ }^{-}\right)$. NMR (DMSO-d $\left.{ }_{6}\right):{ }^{1} \mathrm{H}, \delta 7.58(\mathrm{~s}, 1 \mathrm{H}, \mathrm{C}(3)$ H), $7.85(\mathrm{~d}, 2 \mathrm{H}, \mathrm{C}(5) \mathrm{H}, \mathrm{C}(9) \mathrm{H}), 7.34$ (t, 2H, C(6)H, C(8) $\mathrm{H}), 7.15(\mathrm{~m}, 1 \mathrm{H}, \mathrm{C}(7) \mathrm{H}) ;{ }^{13} \mathrm{C}, \delta 173.9 \mathrm{C}(1), 127.2 \mathrm{C}(2)$, $140.2 \mathrm{C}(3), 133.8 \mathrm{C}(4), 129.9 \mathrm{C}(5)$ and $\mathrm{C}(9), 128.0 \mathrm{C}(6)$ and $\mathrm{C}(8), 128.7 \mathrm{C}(7)$.

$\mathrm{Na}[\mathrm{Au}(\mathrm{fspa})] \cdot \mathrm{H}_{2} \mathrm{O}$ (24). [Au(Hfspa)] (0.10 g, $0.27 \mathrm{mmol}), \mathrm{NaOH}(0.011 \mathrm{~g}, 0.27 \mathrm{mmol}), \mathrm{H}_{2} \mathrm{O}\left(8 \mathrm{~cm}^{3}\right)$, brown solid. Yield: $67 \%$; mp: $210^{\circ} \mathrm{C}$ (Dec.). (Found: C 20.1, H 1.8, S 7.8\%. Calc. for $\mathrm{C}_{7} \mathrm{H}_{8} \mathrm{O}_{5} \mathrm{SAuNa}$ : C 19.8, H $1.9, \mathrm{~S} 7.6 \%)$. IR $\left(\mathrm{cm}^{-1}\right): 1,596 \mathrm{vs}, v_{\text {asym }}\left(\mathrm{CO}_{2}{ }^{-}\right) ; 1,382 \mathrm{~s}$, $v_{\text {sym }}\left(\mathrm{CO}_{2}{ }^{-}\right)$. NMR (DMSO-d $\left.\mathrm{d}_{6}\right):{ }^{1} \mathrm{H}, \delta 7.58(\mathrm{~s}, 1 \mathrm{H}, \mathrm{C}(3)$ H), $7.28(\mathrm{~d}, 1 \mathrm{H}, \mathrm{C}(5) \mathrm{H}), 6.52(\mathrm{t}, 1 \mathrm{H}, \mathrm{C}(6) \mathrm{H}), 7.60(\mathrm{~d}, 1 \mathrm{H}$, $\mathrm{C}(7) \mathrm{H}) ;{ }^{13} \mathrm{C}, \delta 172.4 \mathrm{C}(1), 135.0 \mathrm{C}(2), 121.8 \mathrm{C}(3), 153.2$ $\mathrm{C}(4), 111.8 \mathrm{C}(5), 111.4 \mathrm{C}(6), 141.7 \mathrm{C}(7)$.

$\mathrm{Na}\left[\mathrm{Au}(\right.$ tspa) $] \cdot \mathrm{H}_{2} \mathrm{O}$ (25). [Au(Htspa)] (0.12 g, $\left.0.31 \mathrm{mmol}\right)$, $\mathrm{NaOH}(0.013 \mathrm{~g}, 0.31 \mathrm{mmol}), \mathrm{H}_{2} \mathrm{O}\left(10 \mathrm{~cm}^{3}\right)$, brown solid. Yield: $68 \%$; mp: $221^{\circ} \mathrm{C}$ (Dec.). (Found: C 19.6, H 1.2, S 15.1\%. Calc. for $\mathrm{C}_{7} \mathrm{H}_{6} \mathrm{O}_{3} \mathrm{~S}_{2} \mathrm{AuNa}: \mathrm{C} 19.9, \mathrm{H} 1.4, \mathrm{~S}$ 15.2\%). IR $\left(\mathrm{cm}^{-1}\right)$ : 1,571 vs, $\nu_{\text {asym }}\left(\mathrm{CO}_{2}^{-}\right) ; 1,368 \mathrm{vs,}$ $v_{\text {sym }}\left(\mathrm{CO}_{2}{ }^{-}\right)$. NMR $\left(\right.$DMSO-d $\left._{6}\right):{ }^{1} \mathrm{H}, \delta 7.78(\mathrm{~s}, 1 \mathrm{H}, \mathrm{C}(3)$ H), $7.35(\mathrm{~d}, 1 \mathrm{H}, \mathrm{C}(5) \mathrm{H}), 7.04(\mathrm{t}, 1 \mathrm{H}, \mathrm{C}(6) \mathrm{H}), 7.45(\mathrm{~d}, 1 \mathrm{H}$, $\mathrm{C}(7) \mathrm{H}) ;{ }^{13} \mathrm{C}, \delta 172.6 \mathrm{C}(1), 125.2 \mathrm{C}(2), 135.3 \mathrm{C}(3), 142.2$ $\mathrm{C}(4), 130.7 \mathrm{C}(5), 126.3 \mathrm{C}(6), 125.9 \mathrm{C}(7)$.

$\mathrm{Na}\left[\mathrm{Au}(-o\right.$-pyspa) $] \cdot \mathrm{H}_{2} \mathrm{O}$ (26). [Au(H-o-pyspa)] (0.05 g, $0.13 \mathrm{mmol}), \mathrm{NaOH}(0.005 \mathrm{~g}, 0.13 \mathrm{mmol}), \mathrm{H}_{2} \mathrm{O}\left(5 \mathrm{~cm}^{3}\right)$, brown solid. Yield: $48 \%$; mp: $224^{\circ} \mathrm{C}$. (Found: $\mathrm{C} 22.8$, $\mathrm{H}$ 1.7, S 7.9, N 3.2\%. Calc. for $\mathrm{C}_{8} \mathrm{H}_{7} \mathrm{O}_{3} \mathrm{SNAuNa}$ : C 23.0, $\mathrm{H} 1.7, \mathrm{~S} 7.7, \mathrm{~N} 3.3 \%)$. IR $\left(\mathrm{cm}^{-1}\right): 1,574 \mathrm{vs}, \mathrm{V}_{\text {asym }}\left(\mathrm{CO}_{2}{ }^{-}\right)$; $1,381 \mathrm{vs}, \mathrm{v}_{\mathrm{sym}}\left(\mathrm{CO}_{2}{ }^{-}\right)$. NMR (DMSO-d $\left.\mathrm{d}_{6}\right):{ }^{1} \mathrm{H}, \delta 7.10(\mathrm{~s}$, 1H, C(3)H), 7.58 (d, 1H, C(5)H), 7.81 (pst, 1H, C(6)H), 7.19 (pst, $1 \mathrm{H}, \mathrm{C}(7) \mathrm{H}), 8.56(\mathrm{~d}, 1 \mathrm{H}, \mathrm{C}(8) \mathrm{H}) ;{ }^{13} \mathrm{C}, \delta 172.5$ $\mathrm{C}(1), 135.9 \mathrm{C}(2), 133.4 \mathrm{C}(3), 153.2 \mathrm{C}(4), 139.6 \mathrm{C}(5)$, $29.2 \mathrm{C}(6), 121.6 \mathrm{C}(7), 125.6 \mathrm{C}(8)$.

$\mathrm{Na}[\mathrm{Au}(\mathrm{Clpspa})] \cdot \mathrm{H}_{2} \mathrm{O}$ (27). [Au(HClpspa)] (0.10 g, $0.24 \mathrm{mmol}), \mathrm{NaOH}(0.01 \mathrm{~g}, 0.24 \mathrm{mmol}), \mathrm{H}_{2} \mathrm{O}$ $\left(8 \mathrm{~cm}^{3}\right)$, yellow solid. Yield: $60 \%$; mp: $227^{\circ} \mathrm{C}$. (Found: C 23.8, H 1.4, S 7.0\%. Calc. for $\mathrm{C}_{9} \mathrm{H}_{7} \mathrm{O}_{3} \mathrm{SClAuNa}$ : C 24.0, H 1.6, S 7.1\%). IR $\left(\mathrm{cm}^{-1}\right)$ : 1,589 vs, 1,575 vs, $v_{\text {asym }}\left(\mathrm{CO}_{2}{ }^{-}\right) ; 1,384$ vs, 1,367 vs, $v_{\text {sym }}\left(\mathrm{CO}_{2}{ }^{-}\right)$. NMR (DMSO-d ${ }_{6}$ ): ${ }^{1} \mathrm{H}, \delta 7.70(\mathrm{~s}, 1 \mathrm{H}, \mathrm{C}(3) \mathrm{H}), 7.42(\mathrm{~d}, 1 \mathrm{H}$, $\mathrm{C}(6) \mathrm{H}), 7.19$ (t, 1H, C(7)H), 7.31 (t, 1H, C(8)H), 7.90 $(\mathrm{d}, 1 \mathrm{H}, \mathrm{C}(9) \mathrm{H}) ;{ }^{13} \mathrm{C}, \delta 172.3 \mathrm{C}(1), 127.0 \mathrm{C}(2), 133.2 \mathrm{C}$ (3), $138.1 \mathrm{C}(4), 135.2 \mathrm{C}(5), 129.5 \mathrm{C}(6), 129.9 \mathrm{C}(7)$, $126.2 \mathrm{C}(8), 128.2 \mathrm{C}(9)$. 
$\mathrm{Na}\left[\mathrm{Au}(-o\right.$-mpspa) $] \cdot \mathrm{H}_{2} \mathrm{O}(\mathbf{2 8})$. [Au(H-o-mpspa) $](0.10 \mathrm{~g}$, $0.25 \mathrm{mmol}), \mathrm{NaOH}(0.01 \mathrm{~g}, 0.25 \mathrm{mmol}), \mathrm{H}_{2} \mathrm{O}\left(8 \mathrm{~cm}^{3}\right)$, yellow solid. Yield: $63 \%$; mp: $220^{\circ} \mathrm{C}$ (Dec.). (Found: C 27.1, H 2.3, S 7.2\%. Calc. for $\mathrm{C}_{10} \mathrm{H}_{10} \mathrm{O}_{4} \mathrm{SAuNa}$ : C 26.9, H 2.3, S 7.2\%). IR ( $\left.\mathrm{cm}^{-1}\right): 1,566 \mathrm{vs,}, v_{\text {asym }}\left(\mathrm{CO}_{2}{ }^{-}\right)$; $1,372 \mathrm{vs}, v_{\mathrm{sym}}\left(\mathrm{CO}_{2}{ }^{-}\right)$. NMR (DMSO-d $\left.{ }_{6}\right):{ }^{1} \mathrm{H}, \delta 7.73(\mathrm{~s}$, $1 \mathrm{H}, \mathrm{C}(3) \mathrm{H}), 8.00(\mathrm{~d}, 1 \mathrm{H}, \mathrm{C}(6) \mathrm{H}), 6.89(\mathrm{t}, 1 \mathrm{H}, \mathrm{C}(7) \mathrm{H})$, $7.16(\mathrm{t}, 1 \mathrm{H}, \mathrm{C}(8) \mathrm{H}), 7.91(\mathrm{~d}, 1 \mathrm{H}, \mathrm{C}(9) \mathrm{H}), 3.72(\mathrm{~s}, 3 \mathrm{H}$, $\left.\mathrm{OCH}_{3}\right) ;{ }^{13} \mathrm{C}, \delta 173.3 \mathrm{C}(1), 127.6 \mathrm{C}(2), 137.5 \mathrm{C}(3)$, $125.5 \mathrm{C}(4), 156.4 \mathrm{C}(5), 110.2 \mathrm{C}(6), 130.7 \mathrm{C}(7), 119.3$ $\mathrm{C}(8), 129.2 \mathrm{C}(9), 55.3 \mathrm{C}\left(\mathrm{OCH}_{3}\right)$.

$\mathrm{Na}\left[\mathrm{Au}(-p\right.$-mpspa) $] \cdot \mathrm{H}_{2} \mathrm{O}$ (29). [Au(H-p-mpspa)] $(0.08 \mathrm{~g}, 0.2 \mathrm{mmol}), \mathrm{NaOH}(0.01 \mathrm{~g}, 0.2 \mathrm{mmol}), \mathrm{H}_{2} \mathrm{O}$ $\left(6 \mathrm{~cm}^{3}\right)$, orange solid. Yield: $68 \%$; mp: $209^{\circ} \mathrm{C}$ (Dec.). (Found: C 26.7, H 2.1, S 6.9\%. Calc. for $\mathrm{C}_{10} \mathrm{H}_{10} \mathrm{O}_{4} \mathrm{SAgNa}: \mathrm{C} 26.9, \mathrm{H} 2.3, \mathrm{~S} 7.2 \%$ ). IR $\left(\mathrm{cm}^{-1}\right): 1,570 \mathrm{~s}, v_{\mathrm{asym}}\left(\mathrm{CO}_{2}^{-}\right) ; 1,383 \mathrm{~s}, \gamma_{\mathrm{sym}}\left(\mathrm{CO}_{2}{ }^{-}\right)$. NMR (DMSO-d $\mathrm{d}_{6}$ ): ${ }^{1} \mathrm{H}, \delta 7.56(\mathrm{~s}, 1 \mathrm{H}, \mathrm{C}(3) \mathrm{H}), 7.93$ (d, 2H, C(5)H), (C(9)H), $6.87(\mathrm{~d}, 2 \mathrm{H}, \mathrm{C}(6) \mathrm{H}, \mathrm{C}(8) \mathrm{H})$, $3.72\left(\mathrm{~s}, 3 \mathrm{H}, \mathrm{OCH}_{3}\right) ;{ }^{13} \mathrm{C}, \delta 173.4 \mathrm{C}(1), 115.0 \mathrm{C}(2)$, $134.5 \mathrm{C}(3), 130.4 \mathrm{C}(4), 131.2 \mathrm{C}(5)$ and $\mathrm{C}(9), 113.3$ $\mathrm{C}(6)$ and $\mathrm{C}(8), 158.7 \mathrm{C}(7), 54.9 \mathrm{C}\left(\mathrm{OCH}_{3}\right)$.

$\mathrm{Na}\left[\mathrm{Au}(-o\right.$-hpspa) $] \cdot \mathrm{H}_{2} \mathrm{O}$ (30). [Au(H-o-hpspa)] (0.06 g, $0.15 \mathrm{mmol}), \mathrm{NaOH}(0.006 \mathrm{~g}, 0.15 \mathrm{mmol})$, $\mathrm{H}_{2} \mathrm{O}\left(5 \mathrm{~cm}^{3}\right)$, orange solid. Yield: $66 \%$; mp: $225^{\circ} \mathrm{C}$ (Dec.). (Found: C 24.7, H 1.8, S 7.0\%. Calc. for $\mathrm{C}_{9} \mathrm{H}_{8} \mathrm{O}_{4} \mathrm{SAuNa}$ : C 25.0, H 1.9, S 7.4\%). IR $\left(\mathrm{cm}^{-1}\right)$ : 1,566 vs, $v_{\text {asym }}\left(\mathrm{CO}_{2}{ }^{-}\right) ; 1,363$ vs, $v_{\text {sym }}\left(\mathrm{CO}_{2}{ }^{-}\right)$. NMR $\left(\mathrm{DMSO}_{\mathrm{d}}\right.$ ): ${ }^{1} \mathrm{H}, \delta 7.84(\mathrm{~s}, 1 \mathrm{H}, \mathrm{C}(3) \mathrm{H}), 9.63(\mathrm{~s}, 1 \mathrm{H}$, $\mathrm{C}(5) \mathrm{OH}), 6.79(\mathrm{~m}, 2 \mathrm{H}, \mathrm{C}(6) \mathrm{H}$ and $\mathrm{C}(8) \mathrm{H}), 7.00$ (pst, $1 \mathrm{H}, \mathrm{C}(7) \mathrm{H}), 8.10(\mathrm{~d}, 1 \mathrm{H}, \mathrm{C}(9) \mathrm{H}) ;{ }^{13} \mathrm{C}$, \& $173.6 \mathrm{C}(1), 123.9 \mathrm{C}(2), 132.8 \mathrm{C}(3), 155.3 \mathrm{C}(5)$, $114.8 \mathrm{C}(6), 130.4 \mathrm{C}(7), 118.0 \mathrm{C}(8), 129.8 \mathrm{C}(9)$.

$\mathrm{Na}\left[\mathrm{Au}(-p\right.$-hpspa) $] \cdot \mathrm{H}_{2} \mathrm{O}$ (31). [Au(H-p-hpspa)] (0.08 g, $0.2 \mathrm{mmol}), \mathrm{NaOH}(0.008 \mathrm{~g}, 0.2 \mathrm{mmol}), \mathrm{H}_{2} \mathrm{O}\left(6 \mathrm{~cm}^{3}\right)$, orange solid. Yield: $60 \%$; $\mathrm{mp}: 217^{\circ} \mathrm{C}$ (Dec.). (Found: C 24.8, H 2.1, S 7.2\%. Calc. for $\mathrm{C}_{9} \mathrm{H}_{8} \mathrm{O}_{4} \mathrm{SAuNa}$ : C 25.0, $\mathrm{H} 1.9$, S 7.4\%). IR $\left(\mathrm{cm}^{-1}\right)$ : 1,563 vs, $V_{\text {asym }}\left(\mathrm{CO}_{2}^{-}\right)$; $1,360 \mathrm{vs}, v_{\mathrm{sym}}\left(\mathrm{CO}_{2}{ }^{-}\right)$. NMR (DMSO-d $\left.\mathrm{d}_{6}\right):{ }^{1} \mathrm{H}, \delta 7.56$ (s, 1H, C(3)H), 7.87 (d, 2H, C(5)H, C(9)H), 6.71 (d, $2 \mathrm{H}, \mathrm{C}(6) \mathrm{H}, \mathrm{C}(8) \mathrm{H}), 9.50$ (s, $1 \mathrm{H}, \mathrm{C}(7) \mathrm{OH}) ;{ }^{13} \mathrm{C}, \delta 172.6$ $\mathrm{C}(1), 122.2 \mathrm{C}(2), 127.9 \mathrm{C}(4), 131.5 \mathrm{C}(5)$ and $\mathrm{C}(9)$, $114.8 \mathrm{C}(6)$ and $\mathrm{C}(8), 156.3 \mathrm{C}(7)$.

$\mathrm{Na}\left[\mathrm{Au}(-\right.$ diBr-o-hpspa) $] \cdot \mathrm{H}_{2} \mathrm{O}$ (32). [Au(H-diBr-ohpspa)] (0.05 g, $0.09 \mathrm{mmol}), \mathrm{NaOH}(0.007 \mathrm{~g}$, $0.09 \mathrm{mmol}), \mathrm{H}_{2} \mathrm{O}\left(5 \mathrm{~cm}^{3}\right)$, yellow solid. Yield: $48 \%$; mp: $219^{\circ} \mathrm{C}$. (Found: C $17.9, \mathrm{H} 1.1, \mathrm{~S} 5.2 \%$. Calc. for $\mathrm{C}_{9} \mathrm{H}_{6} \mathrm{O}_{4} \mathrm{SBr}_{2} \mathrm{AuNa}$ : C 18.3, H 1.0, S 5.4\%). IR $\left(\mathrm{cm}^{-1}\right)$ : 1,565 vs br, $v_{\text {asym }}\left(\mathrm{CO}_{2}^{-}\right) ; 1,361$ vs br, $v_{\text {sym }}\left(\mathrm{CO}_{2}^{-}\right)$. NMR (DMSO-d $\left.\mathrm{d}_{6}\right):{ }^{1} \mathrm{H}, \delta 7.90(\mathrm{~s}, 1 \mathrm{H}, \mathrm{C}(3) \mathrm{H}), 7.75(\mathrm{~d}$, $1 \mathrm{H}, \mathrm{C}(7) \mathrm{H}), 7.20(\mathrm{~d}, 1 \mathrm{H}, \mathrm{C}(9) \mathrm{H}) ;{ }^{13} \mathrm{C}, \delta 172.8 \mathrm{C}(1)$, $125.6 \mathrm{C}(2), 133.8 \mathrm{C}(3), 126.3 \mathrm{C}(4), 155.6 \mathrm{C}(5), 115.9$ $\mathrm{C}(6), 137.2 \mathrm{C}(7), 112.5 \mathrm{C}(8), 130.2 \mathrm{C}(9)$.
$\mathrm{Na}[\mathrm{Au}(\mathrm{cpa})] \cdot \mathrm{H}_{2} \mathrm{O}(\mathbf{3 3})$. [Au(Hcpa)] $(0.1 \mathrm{~g}, 0.28 \mathrm{mmol})$, $\mathrm{NaOH}(0.012 \mathrm{~g}, 0.28 \mathrm{mmol}), \mathrm{H}_{2} \mathrm{O}\left(8 \mathrm{~cm}^{3}\right)$, brown solid. Yield: $68 \%$; mp: $222^{\circ} \mathrm{C}$. (Found: C 21.5, H 2.8, S 8.2\%. Calc. for $\mathrm{C}_{7} \mathrm{H}_{10} \mathrm{O}_{3} \mathrm{SAuNa}$ : C 21.3, H 2.6, S 8.1\%). IR $\left(\mathrm{cm}^{-1}\right): 1,570 \mathrm{vs}, v_{\text {asym }}\left(\mathrm{CO}_{2}{ }^{-}\right) ; 1,387$ vs br, $v_{\text {sym }}\left(\mathrm{CO}_{2}{ }^{-}\right)$. NMR $\left(\right.$DMSO-d $\left._{6}\right):{ }^{1} \mathrm{H}, \delta 2.65(\mathrm{~m}, 2 \mathrm{H}$, $\left.\mathrm{C}(4) \mathrm{H}_{2}\right), 1.56\left(\mathrm{~m}, \mathrm{C}(5) \mathrm{H}_{2}\right), 1.56\left(\mathrm{~m}, \mathrm{C}(6) \mathrm{H}_{2}\right), 2.36$ (m, $\left.2 \mathrm{H}, \mathrm{C}(7) \mathrm{H}_{2}\right) ;{ }^{13} \mathrm{C}, \delta 172.0 \mathrm{C}(1), 129.6 \mathrm{C}(2), 160.3$ $\mathrm{C}(3), 38.2 \mathrm{C}(4), 26.1 \mathrm{C}(5), 24.8 \mathrm{C}(6), 36.0 \mathrm{C}(7)$.

\section{Results and discussion}

Synthesis and characterization

Complexes were prepared as described in the "Experimental" section. The $[\mathrm{Au}(\mathrm{HL})]$ complexes were obtained in high yields (close to $80 \%$ in most cases), whereas the reactions that afforded $[\mathrm{HQ}][\mathrm{Au}(\mathrm{L})]$ and $\mathrm{Na}[\mathrm{Au}(\mathrm{L})] \cdot \mathrm{H}_{2} \mathrm{O}$ gave lower yields. The three types of compound differ in solubility. The [HQ] $[\mathrm{Au}(\mathrm{L})]$ complexes are soluble in ethanol, methanol, acetone, chloroform and DMSO, the $\mathrm{Na}[\mathrm{Au}(\mathrm{L})] \mathrm{H}_{2} \mathrm{O}$ complexes are soluble in water and DMSO, and the $[\mathrm{Au}(\mathrm{HL})]$ complexes are only soluble in DMSO.

The IR spectra of $[\mathrm{Au}(\mathrm{HL})]$ complexes do not show the $v(\mathrm{SH})$ band present at around $2,550 \mathrm{~cm}^{-1}$ in the spectra of the free ligands. Furthermore, the vibrations of the $\mathrm{COOH}$ group are slightly shifted from their positions in the spectra of the free ligands $[14,15]$. These features suggest that, as in other complexes in which the $\mathrm{COOH}$ group is present, this group is not deprotonated and remains uncoordinated, with the complexes probably being polymeric species supported by $\mathrm{Au}-\mathrm{S}$ bonds, as previously suggested for equivalent silver complexes [14, 15, 39, 40].

In the case of $[\mathrm{HQ}][\mathrm{Au}(\mathrm{L})]$, the common features for the complexes are the absence of the $v(\mathrm{SH})$ band and of the bands due to the $\mathrm{COOH}$ group; both of these observations are consistent with the bideprotonation of the ligand in all cases. The existence of the diisopropylammonium cation is confirmed by the presence, at around $1,600 \mathrm{~cm}^{-1}$, of a band due to the $\mathrm{NH}_{2}{ }^{+}$group [41], which was previously identified in equivalent complexes with the same ligands $[14,15]$. The $v_{\text {asym }}\left(\mathrm{CO}_{2}{ }^{-}\right)$and $v_{\text {sym }}\left(\mathrm{CO}_{2}^{-}\right)$bands are located in similar positions in the spectra of all the complexes, suggesting the same coordination mode for the carboxylate group, which, in all cases, acts as a monodentate group that is hydrogen bonded to the HQ cations [14, 15, 42]

The IR spectra of $\mathrm{Na}[\mathrm{Au}(\mathrm{L})] \cdot \mathrm{H}_{2} \mathrm{O}$ complexes do not contain the $v(\mathrm{SH})$ band or the bands due to the $\mathrm{COOH}$ group. The positions of the $v_{\text {asym }}\left(\mathrm{CO}_{2}{ }^{-}\right)$and $v_{\text {sym }}\left(\mathrm{CO}_{2}{ }^{-}\right)$ bands are again similar in all of the complexes and these positions are again compatible with the same kind of 
monodentate coordination mode for the carboxylate group, which, in these cases, can be hydrogen bonded to the $\mathrm{H}_{2} \mathrm{O}$ molecule instead of the diisopropylammonium cation.

\section{NMR studies}

For complexes of the type $[\mathrm{Au}(\mathrm{HL})](\mathbf{1}-\mathbf{1 1})$, the broad signal at around $13 \mathrm{ppm}$ in the ${ }^{1} \mathrm{H}$ NMR spectra of the ligands persists, an observation consistent with the presence of the protonated $\mathrm{COOH}$ group. This signal is not present in the spectrum of the $[\mathrm{Au}(\mathrm{H}-\mathrm{o}-\mathrm{pyspa})]$ complex, probably due to an interchange with the deuterium of the solvent. In the ${ }^{1} \mathrm{H}$ spectrum of $\mathrm{H}_{2}$-O-pyspa the presence of a broad singlet at $17.85 \mathrm{ppm}$ is consistent with protonation of the pyridine nitrogen, which, together with the presence of only one proton on $\mathrm{C}(3) \mathrm{H}$, suggests that this compound is in the thione form in solution and not in the enethiol form. Coordination to gold causes significant changes in the ${ }^{1} \mathrm{H}$ NMR spectrum; the signal attributed to $\mathrm{N}-\mathrm{H}$ does not appear for the complex, a fact that reflects deprotonation of this group and the evolution of the ligand to the thiol form. The ${ }^{13} \mathrm{C}$ NMR spectra of these complexes show the $C(3)$ signal shifted to higher field with respect to that in the free ligand $[14,15]$, suggesting that the S-coordination found in the solid state, as in other complexes with these ligands [43, 44], is retained in solution.

For $[\mathrm{HQ}][\mathrm{Au}(\mathrm{L})](\mathbf{1 2 - 2 2})$ and $\mathrm{Na}[\mathrm{Au}(\mathrm{L})] \cdot \mathrm{H}_{2} \mathrm{O}(\mathbf{2 3}-\mathbf{3 3})$ compounds the ${ }^{1} \mathrm{H}$ NMR spectra show a shift in the ligand $\mathrm{C}$ (3)H signal to higher field on complexation, which again suggests the persistence of the S-Au bond in solution; the disappearance of the broad signal located at around $13 \mathrm{ppm}$ in the spectra of each free acid evidences the deprotonation of the $\mathrm{COOH}$ group in the complexes. The persistence of the S-coordination was confirmed by the shift in the $\mathrm{C}(3)$ signal in the ${ }^{13} \mathrm{C}$ NMR spectra. In these spectra, the $\mathrm{C}(1)$ peaks are in positions close to those found in compounds with a coordinated carboxylate group $[45,46]$ and, in particular, in the equivalent silver complexes $[14,15]$.

\section{Antimicrobial activity}

Antibacterial and antifungal activities are listed in Tables 1 and 2 , as estimated by minimum inhibitory concentration (MIC; microgrammes per millilitre) and minimum bactericidal concentration (MBC; microgrammes per millilitre). Remarkable activity was not exhibited by either diisopropylammonium chloride [42], the ligands or complexes of the type $[\mathrm{Au}(\mathrm{HL})](\mathbf{1}-11)$, which in the case of the complexes can be attributed to the low solubility.

The majority of these new complexes showed better activity against the Gram $(+)$ bacteria $S$. aureus and $B$. subtilis than against the Gram (-) E. coli and P. aeruginosa (the lower activity is shown against this latter bacterium even though the values are similar to those for E. coli). However, there are differences between the two classes of compounds and also between the compounds included in the $[\mathrm{HQ}][\mathrm{Au}(\mathrm{L})]$ or $\mathrm{Na}[\mathrm{Au}(\mathrm{L})] \cdot \mathrm{H}_{2} \mathrm{O}$ classes.

Among the HQ derivatives, which in general are more active than the $\mathrm{Na}$ derivatives, $[\mathrm{HQ}][\mathrm{Au}(\mathrm{fspa})](\mathbf{1 3})$, [HQ] [Au(-o-pyspa)] (15) and [HQ][Au(-diBr-o-hpspa)] (21) show higher activity against the Gram $(+)$ bacteria; however, the wider spectrum of activity within this class corresponds to $[\mathrm{HQ}][\mathrm{Au}(\mathrm{tspa})](\mathbf{1 4})$, which shows significant activity against the assayed Gram (+) and Gram (-) bacteria.

Among the Na derivatives, the worst values were measured for $\mathrm{Na}[\mathrm{Au}(\mathrm{cpa})] \cdot \mathrm{H}_{2} \mathrm{O}$ (33), which showed a low activity against all the tested bacteria, whereas $\mathrm{Na}[\mathrm{Au}(\mathrm{Clpspa})] \cdot \mathrm{H}_{2} \mathrm{O}$ (27) showed significant activity against all the bacteria; furthermore, $\mathrm{Na}[\mathrm{Au}(\mathrm{pspa})] \cdot \mathrm{H}_{2} \mathrm{O}(\mathbf{2 3})$ showed better activity against the $\mathrm{Gram}(-)$ bacteria and $\mathrm{Na}\left[\mathrm{Au}(-o-\right.$ hpspa) $] \cdot \mathrm{H}_{2} \mathrm{O}(\mathbf{3 0})$ and $\mathrm{Na}$ $[\mathrm{Au}(-\mathrm{diBr}-o-\mathrm{hpspa})] \cdot \mathrm{H}_{2} \mathrm{O}(\mathbf{3 2})$ showed good values against the two Gram $(+)$ bacteria and $E$. coli.

In an attempt to assess the bactericidal or bacteriostatic activity of these compounds, we also determined the MBC values for most of the synthesised compounds (Table 2). In the range of concentrations studied, we found bactericidal activity for some complexes and, as can be seen in Table 2, this activity is particularly relevant against $E$. coli and B. subtilis.

In the first case, among the HQ complexes, [HQ][Au(pspa)] (12), [HQ][Au(fspa)] (13), [HQ][Au(-o-hpspa)] (19) and [HQ] [Au(-p-hpspa)] (20) showed only bacteriostatic activity. The complexes [HQ][Au(tspa)] (14), [HQ][Au(-o-pyspa)] (15), [HQ][Au(Clpspa)] (16), [HQ][Au(-diBr-o-hpspa)] (21) and $[\mathrm{HQ}][\mathrm{Au}(\mathrm{cpa})](\mathbf{2 2})$ showed bactericidal activity but only at concentrations higher than those at which the initial growth inhibition was observed; for [HQ][Au(-o-mpspa)] (17) and $[\mathrm{HQ}][\mathrm{Au}(-p$-mpspa) $](\mathbf{1 8})$, the bacteriostatic and bactericidal activity was observed at the same concentration.

Bactericidal activity was not observed for the $\mathrm{Na}$ complexes $\mathrm{Na}\left[\mathrm{Au}(\right.$ fspa) $] \cdot \mathrm{H}_{2} \mathrm{O}(\mathbf{2 4}), \mathrm{Na}[\mathrm{Au}(\mathrm{tspa})] \cdot \mathrm{H}_{2} \mathrm{O}$ (25), $\mathrm{Na}$ $[\mathrm{Au}(-o-\mathrm{mpspa})] \cdot \mathrm{H}_{2} \mathrm{O}(\mathbf{2 8}), \mathrm{Na}\left[\mathrm{Au}(-o\right.$-hpspa) $] \cdot \mathrm{H}_{2} \mathrm{O}(\mathbf{3 0}), \mathrm{Na}$ $\left[\mathrm{Au}(-p\right.$-hpspa) $] \cdot \mathrm{H}_{2} \mathrm{O}(\mathbf{3 1}), \mathrm{Na}\left[\mathrm{Au}(-\right.$ diBr-o-hpspa) $] \cdot \mathrm{H}_{2} \mathrm{O}$ (32) and $\mathrm{Na}[\mathrm{Au}(\mathrm{cpa})] \cdot \mathrm{H}_{2} \mathrm{O}(\mathbf{3 3})$, whereas this activity was observed for $\mathrm{Na}[\mathrm{Au}(\mathrm{pspa})] \cdot \mathrm{H}_{2} \mathrm{O}$ (23), $\mathrm{Na}\left[\mathrm{Au}(-o\right.$-pyspa) $] \cdot \mathrm{H}_{2} \mathrm{O}$ (26), $\mathrm{Na}[\mathrm{Au}(\mathrm{Clpspa})] \cdot \mathrm{H}_{2} \mathrm{O}\left(\right.$ 27) and $\mathrm{Na}\left[\mathrm{Au}(-p\right.$-mpspa) $] \cdot \mathrm{H}_{2} \mathrm{O}$ (29) at slightly higher concentrations than those at which the bacteriostatic activity was observed.

The wider expression of activity over the range of concentrations tested was identified against $B$. subtilis. With the exception of $\mathrm{Na}[\mathrm{Au}(\mathrm{fspa})] \cdot \mathrm{H}_{2} \mathrm{O}(\mathbf{2 4}), \mathrm{Na}\left[\mathrm{Au}(-o\right.$-mpspa) $] \cdot \mathrm{H}_{2} \mathrm{O}$ (28) and $\mathrm{Na}[\mathrm{Au}(\mathrm{cpa})] \cdot \mathrm{H}_{2} \mathrm{O}(\mathbf{3 3})$, all of the complexes showed bactericidal activity - albeit at higher concentrations than those at which bacteriostatic activity was observed. In any case, none of the microorganisms tested showed tolerance to these products because the MIC/MBC ratio was less than 32 in all cases. 
Table 1 Antimicrobial activities (MICs) of the complexes

\begin{tabular}{|c|c|c|c|c|c|c|}
\hline Compound & E. coli & S. aureus & B. subtilis & P. aeruginosa ATCC 27853 & P. aeruginosa Resistant & C. albicans \\
\hline [HQ][Au(pspa)] (12) & 25 & 25 & 25 & 50 & 25 & 50 \\
\hline$[\mathrm{HQ}][\mathrm{Au}(\mathrm{fspa})](\mathbf{1 3})$ & 100 & $<6.25$ & $<6.25$ & 100 & 100 & 50 \\
\hline$[\mathrm{HQ}][\mathrm{Au}(\mathrm{tspa})](\mathbf{1 4})$ & 25 & 12.5 & 25 & 12.5 & 12.5 & 100 \\
\hline [HQ][Au(-o-pyspa)] (15) & 50 & 12.5 & $<6.25$ & 25 & 50 & 200 \\
\hline [HQ][Au(Clpspa)] (16) & 50 & 12.5 & 12.5 & 50 & 50 & 25 \\
\hline$[\mathrm{HQ}][\mathrm{Au}(-o-\mathrm{mpspa})](\mathbf{1 7})$ & 50 & 12.5 & 25 & 25 & 25 & 50 \\
\hline$[\mathrm{HQ}][\mathrm{Au}(-p$-mpspa) $]$ (18) & 50 & 12.5 & 25 & 25 & 25 & 50 \\
\hline [HQ][Au(-o-hpspa)] (19) & 50 & 50 & 25 & 50 & 50 & $>200$ \\
\hline$[\mathrm{HQ}][\mathrm{Au}(-p$-hpspa) $](\mathbf{2 0})$ & 50 & 12.5 & 100 & 50 & 50 & 200 \\
\hline [HQ][Au(-diBr-o-hpspa)] (21) & 50 & 12.5 & $<6.25$ & 50 & 50 & 50 \\
\hline$[\mathrm{HQ}][\mathrm{Au}(\mathrm{cpa})](\mathbf{2 2})$ & 50 & 12.5 & 12.5 & 50 & 50 & 200 \\
\hline $\mathrm{Na}[\mathrm{Au}($ pspa $)] \cdot \mathrm{H}_{2} \mathrm{O}(\mathbf{2 3})$ & 25 & 50 & 50 & 25 & 12.5 & $>200$ \\
\hline $\mathrm{Na}\left[\mathrm{Au}(\right.$ fspa) $] \cdot \mathrm{H}_{2} \mathrm{O}(\mathbf{2 4})$ & 100 & 12.5 & 200 & 100 & 100 & 200 \\
\hline $\mathrm{Na}\left[\mathrm{Au}(\right.$ tspa) $] \cdot \mathrm{H}_{2} \mathrm{O}(\mathbf{2 5})$ & 50 & 25 & 50 & 100 & 100 & 200 \\
\hline $\mathrm{Na}\left[\mathrm{Au}(-o\right.$-pyspa) $] \cdot \mathrm{H}_{2} \mathrm{O}(\mathbf{2 6})$ & 25 & 12.5 & 50 & 100 & 100 & 200 \\
\hline $\mathrm{Na}\left[\mathrm{Au}(\right.$ Clpspa) $] \cdot \mathrm{H}_{2} \mathrm{O}(\mathbf{2 7})$ & 12.5 & 12.5 & 12.5 & 25 & 12.5 & $>200$ \\
\hline $\mathrm{Na}[\mathrm{Au}(-o$-mpspa $)] \cdot \mathrm{H}_{2} \mathrm{O}(\mathbf{2 8})$ & 25 & 25 & 25 & 100 & 100 & 100 \\
\hline $\mathrm{Na}\left[\mathrm{Au}(-p\right.$-mpspa) $] \cdot \mathrm{H}_{2} \mathrm{O}(\mathbf{2 9})$ & 50 & 25 & 50 & 50 & 50 & $>200$ \\
\hline $\mathrm{Na}[\mathrm{Au}(-o-\mathrm{hpspa})] \cdot \mathrm{H}_{2} \mathrm{O}(\mathbf{3 0})$ & $<6.25$ & $<6.25$ & 12.5 & 50 & 50 & 100 \\
\hline $\mathrm{Na}\left[\mathrm{Au}(-p\right.$-hpspa) $] \cdot \mathrm{H}_{2} \mathrm{O}(\mathbf{3 1})$ & 50 & 50 & 25 & 50 & 50 & $>200$ \\
\hline $\mathrm{Na}\left[\mathrm{Au}(-\mathrm{diBr}-o\right.$-hpspa) $] \cdot \mathrm{H}_{2} \mathrm{O}$ (32) & 12.5 & $<6.25$ & $<6.25$ & 50 & 50 & 200 \\
\hline $\mathrm{Na}[\mathrm{Au}(\mathrm{cpa})] \cdot \mathrm{H}_{2} \mathrm{O}(\mathbf{3 3})$ & $>200$ & $>200$ & $>200$ & $>200$ & $>200$ & $>200$ \\
\hline
\end{tabular}

Minimum inhibitory concentration (microgrammes per millilitre)

Only three compounds showed bactericidal activity against S. aureus, and these were $[\mathrm{HQ}][\mathrm{Au}(\mathrm{fspa})](\mathbf{1 3}),[\mathrm{HQ}][\mathrm{Au}$ (-diBr-o-hpspa)] (21) and $\mathrm{Na}\left[\mathrm{Au}\left(-\right.\right.$ diBr-o-hpspa)] $\mathrm{H}_{2} \mathrm{O}$ (32); interestingly, for $[\mathrm{HQ}][\mathrm{Au}(\mathrm{fspa})](\mathbf{1 3})$ and $[\mathrm{HQ}][\mathrm{Au}(-\mathrm{diBr}-o-$ hpspa)] (21), bactericidal activity was detected at low concentrations, indicating a lack of tolerance to these compounds.

Against $P$. aeruginosa, only three HQ derivatives, [HQ][Au(tspa)] (14), [HQ][Au(Clpspa)] (16) and [HQ]
Table $2 \mathrm{MBC}$ values for the complexes
Minimum bactericidal concentration (microgrammes per millilitre)

\begin{tabular}{|c|c|c|c|c|c|}
\hline Compound & E. coli & S. aureus & B. subtilis & $\begin{array}{l}\text { P. aeruginosa } \\
\text { ATCC } 27853\end{array}$ & $\begin{array}{l}\text { P. aeruginosa } \\
\text { Resistant }\end{array}$ \\
\hline$[\mathrm{HQ}][\mathrm{Au}(\mathrm{pspa})](\mathbf{1 2})$ & & & 50 & & \\
\hline$[\mathrm{HQ}][\mathrm{Au}(\mathrm{fspa})](\mathbf{1 3})$ & & 6.25 & 50 & & \\
\hline$[\mathrm{HQ}][\mathrm{Au}(\mathrm{tspa})](\mathbf{1 4})$ & 100 & & 100 & 50 & \\
\hline$[\mathrm{HQ}][\mathrm{Au}(-o-$ pyspa $)](\mathbf{1 5})$ & 100 & & 50 & & \\
\hline [HQ][Au(Clpspa)] (16) & 100 & & 25 & 100 & \\
\hline$[\mathrm{HQ}][\mathrm{Au}(-o$-mpspa) $](\mathbf{1 7})$ & 50 & & 50 & & \\
\hline$[\mathrm{HQ}][\mathrm{Au}(-p$-mpspa) $](\mathbf{1 8})$ & 50 & & 100 & 100 & \\
\hline$[\mathrm{HQ}][\mathrm{Au}(-\mathrm{diBr}-o$-hpspa)] (21) & 100 & 12.5 & 6.25 & & \\
\hline$[\mathrm{HQ}][\mathrm{Au}(\mathrm{cpa})](\mathbf{2 2})$ & 100 & & 50 & & \\
\hline $\mathrm{Na}[\mathrm{Au}(\mathrm{pspa})] \cdot \mathrm{H}_{2} \mathrm{O}(\mathbf{2 3})$ & 50 & & 100 & 100 & 200 \\
\hline $\mathrm{Na}[\mathrm{Au}(\mathrm{tspa})] \cdot \mathrm{H}_{2} \mathrm{O}(\mathbf{2 5})$ & & & 100 & & \\
\hline $\mathrm{Na}[\mathrm{Au}(-o-$-pyspa $)] \cdot \mathrm{H}_{2} \mathrm{O}(\mathbf{2 6})$ & 200 & & 200 & 200 & \\
\hline $\mathrm{Na}[\mathrm{Au}(\mathrm{Clpspa})] \cdot \mathrm{H}_{2} \mathrm{O}(\mathbf{2 7})$ & 50 & & 50 & 100 & 50 \\
\hline $\mathrm{Na}\left[\mathrm{Au}(-p\right.$-mpspa) $] \cdot \mathrm{H}_{2} \mathrm{O}$ (29) & 100 & & 100 & & \\
\hline $\mathrm{Na}\left[\mathrm{Au}(-\right.$ diBr-o-hpspa) $] \cdot \mathrm{H}_{2} \mathrm{O}$ (32) & & 25 & 12.5 & 200 & 200 \\
\hline
\end{tabular}


[Au(-p-mpspa)] (18), and four $\mathrm{Na}$ derivatives, $\mathrm{Na}[\mathrm{Au}$ (pspa) $] \cdot \mathrm{H}_{2} \mathrm{O}$ (23), $\mathrm{Na}\left[\mathrm{Au}(-o\right.$-pyspa) $] \cdot \mathrm{H}_{2} \mathrm{O}$ (26), $\mathrm{Na}[\mathrm{Au}$
(Clpspa) $] \cdot \mathrm{H}_{2} \mathrm{O}$ (27) and $\mathrm{Na}\left[\mathrm{Au}(-\right.$ diBr-o-hpspa) $] \cdot \mathrm{H}_{2} \mathrm{O}$ (32), showed bactericidal activity; the same situation

Table 3 Antimicrobial activities (MICs) of the complexes prepared in this work and other compounds containing $\mathrm{Ag}-\mathrm{S}$ bonds

\begin{tabular}{|c|c|c|c|c|c|c|}
\hline Compound & E. coli & $S$ aureus & B. subtilis & P. aeruginosa & Resistant $P$. aeruginosa & C. albicans \\
\hline$[\mathrm{HQ}][\mathrm{Au}(\mathrm{pspa})](\mathbf{1 2})^{\mathrm{a}}$ & 25 & 25 & 25 & 50 & 25 & 50 \\
\hline$[\mathrm{HQ}][\mathrm{Ag}(\mathrm{pspa})]^{\mathrm{b}}$ & 200 & 100 & 50 & 25 & 25 & 50 \\
\hline$[\mathrm{HQ}][\mathrm{Au}(\mathrm{fspa})](\mathbf{1 3})^{\mathrm{a}}$ & 100 & $<6.25$ & $<6.25$ & 100 & 100 & 50 \\
\hline$[\mathrm{HQ}][\mathrm{Ag}(\mathrm{fspa})]^{\mathrm{b}}$ & 200 & 50 & 50 & 25 & 25 & 50 \\
\hline$[\mathrm{HQ}][\mathrm{Au}(\mathrm{tspa})](\mathbf{1 4})^{\mathrm{a}}$ & 25 & 12.5 & 25 & 12.5 & 12.5 & 100 \\
\hline$[\mathrm{HQ}][\mathrm{Ag}(\mathrm{tspa})]^{\mathrm{b}}$ & $>200$ & 200 & 100 & 100 & 50 & 50 \\
\hline$[\mathrm{HQ}][\mathrm{Au}(-o-$ pyspa $)](\mathbf{1 5})^{\mathrm{a}}$ & 50 & 12.5 & $<6.25$ & 25 & 50 & 200 \\
\hline$[\mathrm{HQ}][\operatorname{Ag}(-o-\text { pyspa })]^{\mathrm{b}}$ & 200 & 200 & 100 & 100 & 100 & 100 \\
\hline$[\mathrm{HQ}][\mathrm{Au}(\mathrm{Clpspa})](\mathbf{1 6})^{\mathrm{a}}$ & 50 & 12.5 & 12.5 & 50 & 50 & 25 \\
\hline$[\mathrm{HQ}][\mathrm{Ag}(\mathrm{Clpspa})]^{\mathrm{c}}$ & 200 & 50 & 50 & 25 & 25 & 100 \\
\hline$[\mathrm{HQ}][\mathrm{Au}(-o-\mathrm{mpspa})](\mathbf{1 7})^{\mathrm{a}}$ & 50 & 12.5 & 25 & 25 & 25 & 50 \\
\hline$[\mathrm{HQ}][\mathrm{Ag}(-o-\mathrm{mpspa})]^{\mathrm{c}}$ & 100 & 50 & 50 & 12.5 & 25 & 25 \\
\hline$[\mathrm{HQ}]\left[\mathrm{Au}(-p\right.$-mpspa) $](\mathbf{1 8})^{\mathrm{a}}$ & 50 & 12.5 & 25 & 25 & 25 & 50 \\
\hline$[\mathrm{HQ}][\mathrm{Au}(-o-\mathrm{hpspa})](\mathbf{1 9})^{\mathrm{a}}$ & 50 & 50 & 25 & 50 & 50 & $>200$ \\
\hline$[\mathrm{HQ}][\operatorname{Ag}(-o-\mathrm{hpspa})]^{\mathrm{c}}$ & 200 & 100 & 25 & 25 & $>200$ & 25 \\
\hline$[\mathrm{HQ}]\left[\mathrm{Au}(-p\right.$-hpspa) $](\mathbf{2 0})^{\mathrm{a}}$ & 50 & 12.5 & 100 & 50 & 50 & 200 \\
\hline$[\mathrm{HQ}][\operatorname{Ag}(-p \text {-hpspa })]^{\mathrm{c}}$ & 200 & 100 & 25 & 25 & $>200$ & 25 \\
\hline$[\mathrm{HQ}]\left[\mathrm{Au}(-\mathrm{diBr}-o\right.$-hpspa) $](\mathbf{2 1})^{\mathrm{a}}$ & 50 & 12.5 & $<6.25$ & 50 & 50 & 50 \\
\hline$[\mathrm{HQ}]\left[\mathrm{Ag}(-\mathrm{diBr}-o \text {-hpspa) }]^{\mathrm{c}}\right.$ & 200 & 100 & 25 & 50 & 25 & 100 \\
\hline$[\mathrm{HQ}][\mathrm{Au}(\mathrm{cpa})](\mathbf{2 2})^{\mathrm{a}}$ & 50 & 12.5 & 12.5 & 50 & 50 & 200 \\
\hline$[\mathrm{HQ}][\mathrm{Ag}(\mathrm{cpa})]^{\mathrm{b}}$ & $>200$ & $>200$ & 100 & 200 & 100 & 200 \\
\hline $\mathrm{Na}[\mathrm{Au}($ pspa $)] \cdot \mathrm{H}_{2} \mathrm{O}(\mathbf{2 3})^{\mathrm{a}}$ & 25 & 50 & 50 & 25 & 12.5 & $>200$ \\
\hline $\mathrm{Na}[\mathrm{Ag}($ pspa $)] \cdot \mathrm{H}_{2} \mathrm{O}^{\mathrm{b}}$ & $>200$ & 25 & 25 & 50 & 25 & 50 \\
\hline $\mathrm{Na}[\mathrm{Au}(\mathrm{fspa})] \cdot \mathrm{H}_{2} \mathrm{O}(\mathbf{2 4})^{\mathrm{a}}$ & 100 & 12.5 & 200 & 100 & 100 & 200 \\
\hline $\mathrm{Na}[\mathrm{Ag}($ fspa $)] \cdot \mathrm{H}_{2} \mathrm{O}^{\mathrm{b}}$ & 100 & 25 & 12.5 & 25 & 6.25 & 50 \\
\hline $\mathrm{Na}[\mathrm{Au}(\mathrm{tspa})] \cdot \mathrm{H}_{2} \mathrm{O}(\mathbf{2 5})^{\mathrm{a}}$ & 50 & 25 & 50 & 100 & 100 & 200 \\
\hline $\mathrm{Na}[\mathrm{Ag}(\mathrm{tspa})] \cdot \mathrm{H}_{2} \mathrm{O}^{\mathrm{b}}$ & 100 & 25 & 25 & 50 & 25 & 100 \\
\hline $\mathrm{Na}[\mathrm{Au}(-o-$ pyspa $)] \cdot \mathrm{H}_{2} \mathrm{O}(\mathbf{2 6})$ & 25 & 12.5 & 50 & 100 & 100 & 200 \\
\hline $\mathrm{Na}[\mathrm{Au}($ Clpspa $)] \cdot \mathrm{H}_{2} \mathrm{O}(27)^{\mathrm{a}}$ & 12.5 & 12.5 & 12.5 & 25 & 12.5 & $>200$ \\
\hline $\mathrm{Na}[\mathrm{Ag}(\mathrm{Clpspa})] \cdot \mathrm{H}_{2} \mathrm{O}^{\mathrm{c}}$ & $>200$ & 12.5 & 12.5 & 50 & 12.5 & $>200$ \\
\hline $\mathrm{Na}[\mathrm{Au}(-o-\mathrm{mpspa})] \cdot \mathrm{H}_{2} \mathrm{O}(\mathbf{2 8})^{\mathrm{a}}$ & 25 & 25 & 25 & 100 & 100 & 100 \\
\hline $\mathrm{Na}[\mathrm{Ag}(-o-\mathrm{mpspa})] \cdot \mathrm{H}_{2} \mathrm{O}^{\mathrm{c}}$ & $>200$ & 12.5 & 12.5 & 50 & 12.5 & 50 \\
\hline $\mathrm{Na}[\mathrm{Au}(-p-\mathrm{mpspa})] \cdot \mathrm{H}_{2} \mathrm{O}(\mathbf{2 9})^{\mathrm{a}}$ & 50 & 25 & 50 & 50 & 50 & $>200$ \\
\hline $\mathrm{Na}[\mathrm{Au}(-o-\mathrm{hpspa})] \cdot \mathrm{H}_{2} \mathrm{O}(\mathbf{3 0})^{\mathrm{a}}$ & $<6.25$ & $<6.25$ & 12.5 & 50 & 50 & 100 \\
\hline $\mathrm{Na}[\mathrm{Ag}(-o-\mathrm{hpspa})] \cdot 2 \mathrm{H}_{2} \mathrm{O}^{\mathrm{c}}$ & 200 & 200 & 100 & 200 & 200 & $>200$ \\
\hline $\mathrm{Na}[\mathrm{Au}(-p$-hpspa $)] \cdot \mathrm{H}_{2} \mathrm{O}(\mathbf{3 1})^{\mathrm{a}}$ & 50 & 50 & 25 & 50 & 50 & $>200$ \\
\hline $\mathrm{Na}[\mathrm{Ag}(-p-\mathrm{hpspa})] \cdot 2 \mathrm{H}_{2} \mathrm{O}^{\mathrm{c}}$ & 100 & $>200$ & 6.25 & 50 & 25 & 50 \\
\hline $\mathrm{Na}\left[\mathrm{Au}(-\right.$ diBr-o-hpspa) $] \cdot \mathrm{H}_{2} \mathrm{O}(\mathbf{3 2})^{\mathrm{a}}$ & 12.5 & $<6.25$ & $<6.25$ & 50 & 50 & 200 \\
\hline $\mathrm{Na}[\mathrm{Ag}(-\mathrm{diBr}-o-\mathrm{hpspa})] \cdot 2 \mathrm{H}_{2} \mathrm{O}^{\mathrm{c}}$ & $>200$ & 50 & 200 & $>200$ & $>200$ & $>200$ \\
\hline $\mathrm{Na}[\mathrm{Au}(\mathrm{cpa})] \cdot \mathrm{H}_{2} \mathrm{O}(\mathbf{3 3})^{\mathrm{a}}$ & $>200$ & $>200$ & $>200$ & $>200$ & $>200$ & $>200$ \\
\hline $\mathrm{Na}[\mathrm{Ag}(\mathrm{cpa})] \cdot \mathrm{H}_{2} \mathrm{O}^{\mathrm{b}}$ & $>200$ & 50 & $>200$ & $>200$ & $>200$ & 50 \\
\hline
\end{tabular}

Minimum inhibitory concentration (microgrammes per millilitre)

${ }^{\mathrm{a}}$ This work

${ }^{\mathrm{b}}$ Ref. [14]

${ }^{\mathrm{c}}$ Ref. [15] 
was found for the resistant strain of this bacterium, but in this case, only $\mathrm{Na}\left[\mathrm{Au}(\right.$ pspa) $] \cdot \mathrm{H}_{2} \mathrm{O}(\mathbf{2 3}), \mathrm{Na}[\mathrm{Au}(\mathrm{Clpspa})] \cdot \mathrm{H}_{2} \mathrm{O}$ (27) and $\mathrm{Na}\left[\mathrm{Au}(-\mathrm{diBr}-\mathrm{O}\right.$-hpspa) $] \cdot \mathrm{H}_{2} \mathrm{O}$ (32) showed bactericidal activity.

The MIC values for the silver complexes shown in Table 3 enable a comparison of the activity between silver and gold complexes that incorporate the same ligand. It is interesting to underline the better activity of some gold compounds against E. coli, a $\operatorname{Gram}(-)$ bacterium that shows a low sensitivity to the previously prepared silver complexes.

The behaviour outlined above, with some exceptions - in particular for the Na derivatives - was also observed against the two $\operatorname{Gram}(+)$ bacteria but it did not persist against $P$. aeruginosa and the carbapenem-resistant $P$. aeruginosa. In these cases, the silver complexes generally showed better values than the gold complexes, as also shown against $C$. albicans.

If we consider the $\mathrm{HQ}$ and $\mathrm{Na}$ derivatives together and analyse the effect of the introduction of $\mathrm{Au}$ instead of Ag while maintaining the same ligand, the most marked effect was observed for -o-hpspa and diBr-o-hpspa, both ligands for which the phenyl group on the $\mathrm{C}(3)$ of the sulfanylpropenoic acid contains an $-\mathrm{OH}$ substituent in the ortho position.

Different activities were also previously found for equivalent $\mathrm{Ag}$ and $\mathrm{Au}$ complexes with P-donor [28], S-donor[13] or N-donor ligands [47]. As an interesting example in the latter case, it was shown [47] that [1benzyl-3-tert-butylimidazol-2-ylidene]AuCl has significant activity against $B$. subtilis and that this activity is higher than that of the equivalent silver complex. Incubation of $B$. subtilis cells with the gold complex increased the cell length, indicating that this compound inhibits bacterial proliferation by blocking cytokinesis. In contrast, only a very small increase in cell length was observed under equivalent incubation with the silver complex. A different mode of antimicrobial action for equivalent silver and gold complexes was also previously proposed by Nomiya et al. [13, 28].

\section{Conclusion}

In summary, compounds of the type [Au(HL)], [HQ][Au(L)] and $\mathrm{Na}[\mathrm{Au}(\mathrm{L})] \cdot \mathrm{H}_{2} \mathrm{O}$ (where $\mathrm{HQ}=$ diisopropylammonium and $\mathrm{H}_{2} \mathrm{~L}$ are various sulfanylcarboxylates) have been synthesised and characterised. The low solubility of the compounds $[\mathrm{Au}(\mathrm{HL})]$ is probably the reason that they are inactive, but several compounds of the types [HQ][Au(L)] and $\mathrm{Na}[\mathrm{Au}(\mathrm{L})] \cdot \mathrm{H}_{2} \mathrm{O}$ show significant activity against the $\operatorname{Gram}(+)$ bacteria $S$. aureus and B. subtilis. The gold compounds generally show better activity than the silver analogues against $S$. aureus and $B$. subtilis, but low sensitivity against $E$. coli, $P$. aeruginosa and $C$. albicans. The results suggest a different mode of antimicrobial action for equivalent silver and gold compounds.

Acknowledgements We thank the Dirección Xeral de I+D, Xunta de Galicia, Spain, for the financial support (IN845B-2010/121).

Open Access This article is distributed under the terms of the Creative Commons Attribution License which permits any use, distribution and reproduction in any medium, provided the original author(s) and source are credited.

\section{References}

1. Clement JL, Jarret PS (1994) Metal-based drugs 1:467-486

2. Silver S (2003) FEMS Microbiol Rev 27:341-353

3. Chopra I (2007) J Antimicrob Chemother 59:587-590

4. Nomiya K, Takahashi S, Noguchi R, Nemoto S, Takayama T, Oda M (2000) Inorg Chem 39:3301-3311

5. Abu-Youssef MAM, Langer V, Öhrström L (2006) Dalton Trans 2542-2550

6. Yesilel OZ, Kastas G, Darcan C, Ilker I, Pasaoglu H, Buyukgunger O (2010) Inorg Chim Acta 363:1849-1858

7. Abu-Youssef MAM, Soliman SM, Langer V, Gohar YM, Hasanen AA, Makhyoun MA, Zaky AH, Öhrström LR (2010) Inorg Chem 49:9788-9797

8. Kasuga NC, Sugie A, Nomiya, K (2004) Dalton Trans 3732-3740

9. Creaven BS, Egan DA, Kavanagh K, McCann M, Mahon M, Noble A, Thati B, Walsh M (2005) Polyhedron 24:949-957

10. Creaven BS, Egan DA, Kavanagh K, McCann M, Noble A, Thati B, Walsh M (2006) Inorg Chim Acta 359:3976-3984

11. Nomiya K, Onoue K, Kondoh Y, Kasuga NC, Nagano H, Oda M, Sakuma S (1995) Polyhedron 14:1359-1367

12. Nomiya K, Onoue K, Kondoh Y, Kasuga NC, Nagano H, Oda M, Sakuma S (1996) Polyhedron 15:2303

13. Nomiya K, Takahashi S, Noguchi R (2000) J Chem Soc, Dalton Trans 2091-2097

14. Barreiro E, Casas JS, Couce MD, Sánchez A, Seoane R, Sordo J, Varela JM, Vázquez-López EM (2007) Dalton Trans 3074-3085

15. Barreiro E, Casas JS, Couce MD, Sánchez A, Seoane R, Sordo J, Varela JM, Vázquez-López EM (2008) Eur J Med Chem 43:24892497

16. Shaw CF III (1999) In: Schmidbaur H (ed) Gold: progress in chemistry, biochemistry and technology. J. Wiley and Sons, Chichester, UK, p 260

17. Parish RV, Cottrill SM (1987) Gold Bull 20:3-12

18. Fricker SP (1996) Gold Bull 29:53-59

19. Eisler R (2003) Inflamm Res 52:487-501

20. Messori L, Marcon G (2004) Met Ions Biol Syst 41:279-304

21. Barreiro E, Casas JS, Couce MD, Gato A, Sánchez S, Sordo J, Varela JM, Vázquez-López EM (2008) Inorg Chem 47:6262-6272

22. Milacic V, Fregona D, Dou QP (2008) Histol Histopathol 23:101108

23. Tiekink ERT (2008) Inflammopharmacology 16:138-142

24. Ott I (2009) Coord Chem Rev 253:1670-1681

25. Navarro M (2009) Coord Chem Rev 253:1619-1626

26. Marques LL, Manzoni de Oliveira G, Schulz Lang E, de Campos MMA, Gris LRS (2007) Inorg Chem Commun 10:1083-1087

27. Noguchi R, Hara A, Sugie A, Nomiya K (2006) Inorg Chem Commun 9:355-359

28. Nomiya K, Noguchi R, Ohsawa K, Tsuda K, Oda M (2000) J Inorg Biochem 78:363-370

29. Nomiya K, Yamamoto S, Noguchi R, Yokohama H, Kasuga NCh, Ohyama K, Kato C (2003) J Inorg Biochem 95:208-220 
30. Elie BT, Levine C, Ubarretxena-Belandia I, Varela-Ramirez A, Aguilera RJ, Ovalle R, Contel M (2009) Eur J Inorg Chem 23:3421-3430

31. Elsome AM, Hamilton-Miller JMT, Brumfitt W, Noble WC (1996) J Antimicrob Chemother 37:911-918

32. Novelli F, Recine M, Sparatore F, Juliano C (1999) Il Farmacol $54: 232-236$

33. Nomiya K, Noguchi R, Shigeta T, Kondoh Y, Tsuda K, Ohsawa K, Kasuga NCh, Oda M (2000) Bull Chem Soc Jpn 73:1143-1152

34. Eiter LC, Hall NM, Day CS, Saluta G, Kucera GL, Bierbach U (2009) J Med Chem 52:6519-6522

35. Fillat MF, Gimeno MC, Laguna A, Latorre E, Ortego L, Villacampa MD (2011) Eur J Inorg Chem 1487-1495

36. Howe BP (1997) Metal-based Drugs 4:273-277

37. Barreiro E, Casas JS, Couce MD, Sánchez A, Sordo J, Varela JM, Vázquez-López EM (2007) Cryst Growth Des 7:1964-1973

38. Koneman EW, Allen SD, Dowell VR Jr, Sommers HM (1979) Color atlas and textbook of diagnostic microbiology. J.B. Lippincott Co., USA, p 321
39. Nomiya K, Kondoh Y, Onoue K, Kasuga NC, Nagano H, Oda M, Sudor T, Sakuma S (1995) J Inorg Biochem 58:255-267

40. Oda M, Itoh H, Sudo T, Sakuma S, Nomiya K, Suzuki Y, Jyonoshita Y, Kikuchi A, Takabatake Y (1995) PTC. WO95/12602

41. Colthup NB, Daly LH, Wilberley SE (1990) Introduction to infrared and Raman spectroscopy, 3rd edn. Academic Press, Inc., San Diego, p 334

42. Casas JS, Castiñeiras A, Couce MD, Jorge ML, Russo U, Sánchez A, Seoane R, Sordo J, Varela JM (2000) Appl Organomet Chem $14: 421-431$

43. Barreiro E, Casas JS, Couce MD, Sánchez A, Sordo J, Varela JM, Vázquez-López EM (2005) Dalton Trans 9:1707-1715

44. Barreiro E, Casas JS, Couce MD, Sánchez A, Sordo J, Varela JM, Vázquez-López EM (2003) Dalton Trans 4754-4781

45. Gajda-Schrantz K, Nagy L, Kuzmann E, Vertes A, Holecek J, Lycka A (1997) J Chem Soc, Dalton Trans 2201-2205

46. Holecek J, Lycka A, Nadvornik M, Handlir K (1991) Collect Czechoslov Chem Commun 56:1908-1915

47. Ray S, Mohan R, Singh JK, Samantaray MK, Shaikh MM, Panda D, Ghosh P (2007) J Am Chem Soc 129:15042-15053 